\title{
Surfactant protein C peptides with salt-bridges ("ion-locks") promote high surfactant activities by mimicking the $\alpha$-helix and membrane topography of the native protein
}

Background: Surfactant protein C (SP-C; 35 residues) in lungs has a cationic N-terminal domain with two cysteines covalently linked to palmitoyls and a C-terminal region enriched in Val, Leu and Ile. Native SP-C shows high surface activity, due to SP-C inserting in the bilayer with its cationic $\mathrm{N}$-terminus binding to the polar headgroup and its hydrophobic $\mathrm{C}$-terminus embedded as a tilted, transmembrane $\alpha$-helix. The palmitoylcysteines in SP-C act as 'helical adjuvants' to maintain activity by overriding the $\beta$-sheet propensities of the native sequences. Objective: We studied SP-C peptides lacking palmitoyls, but containing glutamate and lysine at 4-residue intervals, to assess whether SP-C peptides with salt-bridges ("ion-locks") promote surface activity by mimicking the $\alpha$-helix and membrane topography of native SP-C. Methods: SP-C mimics were synthesized that reproduce native sequences, but without palmitoyls (i.e., SP-Css or SP-Cff, with serines or phenylalanines replacing the two cysteines). lon-lock SP-C molecules were prepared by incorporating single or double Glu-Lys $^{+}$into the parent SP-C's. The secondary structures of SP-C mimics were studied with Fourier transform infrared (FTIR) spectroscopy and PASTA, an algorithm that predicts $\beta$ sheet propensities based on the energies of the various $\beta$-sheet pairings. The membrane topography of SP-C mimics was investigated with orientated and hydrogen/deuterium (H/D) exchange FTIR, and also Membrane Protein Explorer (MPEx) hydropathy analysis. In vitro surface activity was determined using adsorption surface pressure isotherms and captive bubble surfactometry, and in vivo surface activity from lung function measures in a rabbit model of surfactant deficiency. Results: PASTA calculations predicted that the SP-Css and SP-Cff peptides should each form parallel $\beta$-sheet aggregates, with FTIR spectroscopy confirming high parallel $\beta$-sheet with 'amyloid-like' properties. The enhanced $\beta$-sheet properties for SP-Css and SP-Cff are likely responsible for their low surfactant activities in the in vitro and in vivo assays. Although standard ${ }^{12} \mathrm{C}-\mathrm{FTIR}$ study showed that the $\alpha$-helicity of 
these SP-C sequences in lipids was uniformly increased with $\mathrm{Glu}^{-}-\mathrm{Lys}^{+}$insertions, elevated surfactant activity was only selectively observed. Additional results from oriented and H/D exchange FTIR experiments indicated that the high surfactant activities depend on the SP-C ion-locks recapitulating both the $\alpha$-helicity and the membrane topography of native SP-C. SPCss ion-lock 1, an SP-Css with a salt-bridge for a Glu--Lys ${ }^{+}$ion-pair predicted from MPEx hydropathy calculations, demonstrated enhanced surfactant activity and a transmembrane helix simulating those of native SP-C. Conclusion: Highly active SP-C mimics were developed that replace the palmitoyls of SP-C with intrapeptide salt-bridges and represent a new class of synthetic surfactants with therapeutic interest. 


\section{Surfactant Protein C Peptides with Salt-Bridges ("Ion-locks") Promote High 2 Surfactant Activities by Mimicking the $\alpha$-Helix and Membrane Topography of 3 the Native Protein}

4

5

$8{ }^{1}$ Los Angeles Biomedical Research Institute at Harbor-UCLA Medical Center, Torrance,

9 California, United States of America

$10{ }^{2}$ Department of Pediatrics, David Geffen School of Medicine, University of California at Los

11 Angeles, Los Angeles, California, United States of America

$12{ }^{3}$ Department of Medicine, David Geffen School of Medicine, University of California at Los

13 Angeles, Los Angeles, California, United States of America

$14{ }^{4}$ Department of Physiology \& Biophysics, School of Medicine, University of California, Irvine,

15 California, United States of America

$16{ }^{5}$ Department of Pediatrics, University of Rochester, Rochester, New York, United States of

17 America

18

19 Short title: Surfactant Protein C Peptides with Salt-Bridges

20

21 Address for correspondence: Frans J. Walther, M.D., Ph.D., Los Angeles Biomedical Research

22 Institute at Harbor-UCLA Medical Center, 1124 W Carson Street, Torrance, California 90502,

23 United States of America. T (310) 222-8150; F (310) 222-8150; E fjwalther@ucla.edu 


\section{ABSTRACT}

25 Background: Surfactant protein C (SP-C; 35 residues) in lungs has a cationic N-terminal domain with 26 two cysteines covalently linked to palmitoyls and a C-terminal region enriched in Val, Leu and Ile. Native

27 SP-C shows high surface activity, due to SP-C inserting in the bilayer with its cationic N-terminus binding 28 to the polar headgroup and its hydrophobic C-terminus embedded as a tilted, transmembrane $\alpha$-helix. The 29 palmitoylcysteines in SP-C act as 'helical adjuvants' to maintain activity by overriding the $\beta$-sheet 30 propensities of the native sequences.

31 Objective: We studied SP-C peptides lacking palmitoyls, but containing glutamate and lysine at 4-residue 32 intervals, to assess whether SP-C peptides with salt-bridges ("ion-locks") promote surface activity by 33 mimicking the $\alpha$-helix and membrane topography of native SP-C.

34 Methods: SP-C mimics were synthesized that reproduce native sequences, but without palmitoyls (i.e.,

35 SP-Css or SP-Cff, with serines or phenylalanines replacing the two cysteines). Ion-lock SP-C molecules

36 were prepared by incorporating single or double $\mathrm{Glu}^{-}-\mathrm{Lys}^{+}$into the parent SP-C's. The secondary structures of SP-C mimics were studied with Fourier transform infrared (FTIR) spectroscopy and PASTA, an algorithm that predicts $\beta$-sheet propensities based on the energies of the various $\beta$-sheet pairings. The membrane topography of SP-C mimics was investigated with orientated and hydrogen/deuterium (H/D) exchange FTIR, and also Membrane Protein Explorer (MPEx) hydropathy analysis. In vitro surface activity was determined using adsorption surface pressure isotherms and captive bubble surfactometry, and in vivo surface activity from lung function measures in a rabbit model of surfactant deficiency.

43 Results: PASTA calculations predicted that the SP-Css and SP-Cff peptides should each form parallel $\beta$ 44 sheet aggregates, with FTIR spectroscopy confirming high parallel $\beta$-sheet with 'amyloid-like' properties.

45 The enhanced $\beta$-sheet properties for SP-Css and SP-Cff are likely responsible for their low surfactant 46 activities in the in vitro and in vivo assays. Although standard ${ }^{12} \mathrm{C}$-FTIR study showed that the $\alpha$-helicity of 47 these SP-C sequences in lipids was uniformly increased with $\mathrm{Glu}^{-}-\mathrm{Lys}^{+}$insertions, elevated surfactant 48 activity was only selectively observed. Additional results from oriented and H/D exchange FTIR 49 experiments indicated that the high surfactant activities depend on the SP-C ion-locks recapitulating both 50 the $\alpha$-helicity and the membrane topography of native SP-C. SP-Css ion-lock 1, an SP-Css with a salt- 
51 bridge for a $\mathrm{Glu}^{-}-\mathrm{Lys}^{+}$ion-pair predicted from MPEx hydropathy calculations, demonstrated enhanced

52 surfactant activity and a transmembrane helix simulating those of native SP-C.

53 Conclusion: Highly active SP-C mimics were developed that replace the palmitoyls of SP-C with

54 intrapeptide salt-bridges and represent a new class of synthetic surfactants with therapeutic interest.

55

56 Keywords: Surfactant Protein C; synthetic surfactant; protein synthesis; salt-bridges; Fourier Transform

57 InfraRed spectroscopy; PASTA algorithm; Membrane Protein Explorer program; surface activity;

58 surfactant-deficient rabbit model 
INTRODUCTION

Lung surfactant is a mixture of lipids and proteins that is critical for normal breathing due to its

61 ability to prevent alveolar collapse during expiration by reducing alveolar surface tension to extremely

62 low values. Surfactant is synthesized and secreted into the alveolar fluid by alveolar type II cells and

63 consists of approximately 80\% phospholipids, 10\% neutral lipids, and 10\% proteins (Goerke, 1998).

64 Although dipalmitoyl phosphatidylcholine and phosphatidylglycerol constitute the principal phospholipid

65 components in lung surfactant, its biophysical activities largely depend on the presence of the hydrophobic

66 surfactant protein B (SP-B) and, to a lesser degree, the extremely hydrophobic surfactant protein C (SP-C)

67 (Walther et al., 2007a). Pioneering surfactant therapy using bovine or porcine lung extracts, which consist

68 of polar lipids and native SP-B and SP-C, has greatly improved the outcome of premature infants with

69 neonatal respiratory distress syndrome (NRDS) (Polin \& Carlo, 2014). On the other hand, replacement

70 surfactant therapies using animal preparations have not been successful in clinical trials of pediatric and

71 adult patients with acute lung injury (ALI) and acute respiratory distress (ARDS) (Brower \& Fessler,

72 2011; Willson et al., 2013). A major goal of current research is to replace animal-derived formulations for

73 the various surfactant deficiencies with total synthetics consisting of SP-B and SP-C mimics, produced by

74 recombinant technology or peptide synthesis, and selected synthetic lipids (Curstedt, Calkovska \&

75 Johansson, 2013; Walther et al., 2005, 2007b, 2010 \& 2014; Spragg et al., 2011; Almlen et al., 2010).

SP-C is a short (35 amino acids; MW of $4.2 \mathrm{kDa}$ in humans) protein that is highly enriched in

valine, leucine and isoleucine residues, making it much smaller and more hydrophobic than SP-B (79

78 amino acids; MW of $8.7 \mathrm{kDa}$ ) (Walther et al., 2007a). Mature SP-C has an N-terminus with a pair of

79 vicinal cysteine residues that are covalently linked to palmitoyl moieties via thioester bonds (Fig. 1),

80 thereby producing a true proteolipid (Curstedt et al., 1990). The only exception to this vicinal cysteine

81 pairing is found in dog SP-C, which has one palmitate substituted by a phenylalanine (Fig. 1) (Johansson

82 et al., 1991). The palmitoylated groups in SP-C are adjacent to a short polar, N-terminal segment,

83 characterized by cationic residues such as lysine and arginine (Fig. 1). Native SP-C is polymorphic and

84 often has truncations in the N-terminal domain varying from one to three residues. The polar N-terminal 
region of SP-C (residues $\sim 1-8$ ) is followed by a highly conserved and hydrophobic C-terminal sequence enriched in polyvaline that spans for $\sim 26$ residues (9-34) to close to the C-terminus (Fig. 1). Prior Fourier transform infrared (FTIR) spectroscopy showed that native dipalmitoylated SP-C was primarily $\alpha$-helical in surfactant lipid bilayers. Moreover, FTIR spectra indicated that SP-C's long molecular axis was deeply embedded as a transmembrane helix using, hydrogen/deuterium (H/D) exchange and at a slight tilt to the membrane normal with orientation-dependent FTIR analysis (Vandenbussche et al., 1992; Pastrana, Maulone \& Mendelsohn, 1991). Subsequent two-dimensional nuclear magnetic resonance (2D-NMR) of porcine SP-C in a lipid-mimic indicated an unstructured N-terminus (residues $\sim 1-8$ ), while residues $\sim 9-34$ formed an $\alpha$-helix in the mid- and C-terminal regions (PDB accession code: 1SPF) (Johansson et al., 1994). Additional physical studies indicated that the cationic N-terminal domain of SP-C is limited to the surface of phospholipid bilayers and has a higher affinity for anionic than zwitterionic lipids (Plasencia et al., 2004). In agreement with these experimental results, recent Molecular Dynamics simulations predicted that SP-C and its attached palmitoyls penetrate deeply into lipid monolayers (Baoukina \& Tieleman, 2010; Duncan \& Larson, 2010). MD simulations also confirmed that SP-C embeds into bilayers with its helical axis and palmitoyl groups nearly parallel to the fatty acyl chains and its cationic N-terminal region restricted to the membrane headgroup (Baoukina \& Tieleman, 2010). surfactant activities in the human lung (Walther et al., 2007a; Johansson, 1998). With or without the covalent-linked palmitoyl groups, SP-C was shown using various physical techniques to stabilize

104 phospholipid bilayer ensembles that remain attached to the monolayer at the air-water interface. Such

105 adducts may constitute a surfactant lipid 'reservoir' enriched in SP-C that maintains the lipid monolayer

106 throughout the respiration cycle (von Nahmen et al., 1997; Kramer et al., 2000; Ding et al., 2001;

107 Takamoto et al., 2001). The presence of SP-C in lung surfactant also enhances the resistance to surfactant

108 flow by increasing surface viscosity (Alonso \& Zasadzinski, 2004). SP-C plays a lesser role in lung

109 surfactant activities than SP-B. Complete absence of SP-B is lethal in human newborns and animals

110 (Clark et al., 1995), but animals with an SP-C mutation are viable with a disease that disrupts lung 
111 development (Bridges, 2003) and increases susceptibility to infection (Bridges et al, 2006). Thus, a total

112 synthetic replacement that successfully treats patients with surfactant deficiencies is likely to include

113 mimics of both SP-B and SP-C proteins (Curstedt, Calkovska \& Johansson, 2013; Almlen et al., 2010;

114 Walther et al., 2014).

115 Prior investigations indicate that the two palmitoylated cysteine moieties of SP-C play critical

116 roles in the structural and functional properties of this surfactant protein. These neighboring palmitoyl

117 groups stabilize the $\alpha$-helix of SP-C (Vandenbussche et al., 1992; Pastrana, Maulone \& Mendelsohn, 1991;

118 Johansson et al., 1994; Wang et al., 1996; Johansson et al., 1995; Dluhy et al., 2003) and minimize the

119 formation of $\beta$-sheet oligomers (Johansson, 1998; Gustafsson et al., 1999), optimize surface active

120 interactions with lipids (Wang et al., 1996; Baumgart et al., 2010), and may induce a coupling between

121 neighboring layers and even trigger a stacking of bilayers (Baumgart et al., 2010; Na Nakorn et al., 2007).

122 Although SP-C exists primarily as a dipalmitoylated, $\alpha$-helical monomer in bovine lung lavage, there is

123 also a minor dimeric component $(\sim 10 \%)$ that is depalmitoylated (i.e., lacks the two palmitoyls) and

124 exhibits predominantly intermolecular $\beta$-sheet and abnormal in vitro surfactant activities (Baatz et al.,

125 1992). Analogous oligomers of non-palmitoylated SP-C are also connected to pulmonary alveolar

126 proteinosis. Voss et al. (Voss et al., 1992) earlier reported that partially, or even completely,

127 depalmitoylated SP-C, was present in the bronchoalveolar fluid of patients with pulmonary alveolar

128 proteinosis, and suggested that this pathological modification may reduce surfactant function. This

129 hypothesis is also supported by the diminished in vitro surfactant activities seen with chemically

130 deacylated SP-C (Wang et al., 1996), and also the high $\beta$-sheet observed with non-palmitoylated SP-C

131 fibrils using FTIR spectroscopy (Dluhy et al., 2003). More recently, SP-C, with or without palmitoyls, has

132 been identified in the insoluble aggregates of pulmonary alveolar proteinosis fluid and shows

133 characteristic amyloid properties such as Congo red staining and alveolar fibril formation on electron

134 microscopy (Gustafsson et al., 1999). Mutations in the SP-C gene have been associated with familial

135 interstitial lung disease (Nogee et al., 2001) and accumulation and misrouting of incorrectly folded

136 proteins (Whitsett, 2002). Direct observations of amyloid deposits containing mature SP-C in lung tissue 
137 from interstitial lung disease patients with mutations in the BRICHOS domain strongly support this

138 association (Willander et al., 2012). Altogether the various structural studies demonstrate that SP-C is

139 metastable in lipid environments and may partially convert from predominately $\alpha$-helix into insoluble and

140 inactive 'amyloid-like' fibrils consisting of $\beta$-sheet.

141 An early approach to developing SP-C mimics for surfactant replacement therapy was to largely

142 reproduce the sequences of native SP-C using chemically synthesized or recombinant molecules. These

143 first generation SP-C mimics primarily follow the native sequence, but typically omit the covalently

144 attached palmitoyl groups to simplify peptide synthesis and purification. For example, the 34-residue SP-

145 Css mimic principally uses the human SP-C sequence as a template but substitutes the palmitoylcysteine

146 groups with serine residues (Fig. 1). The serine replacements approximate the stereochemistry, hydropathy

147 and net charge of native cysteine without being subject to oxidative side-reactions, and thus SP-Css may

148 be a more stable mimic of depalmitoylated SP-C. The 34-residue SP-Cff (Fig. 1) also uses human SP-C as

149 a template but the palmitoylcysteine groups are replaced with phenylalanines to simulate dipalmitoylated

150 SP-C. Because one of the palmitates in dog SP-C is replaced by phenylalanine (Fig. 1) (Johansson et al.,

151 1991), SP-Cff might exhibit high surfactant activity because its two phenylalanines have bulky

152 hydrophobic side chains that may reproduce the insertion of palmitoyls into the hydrophobic interior of

153 surfactant lipid monolayers/bilayers. Unfortunately, research on chemically synthesized, 35-residue SP-

154 Css or SP-Cff indicated diminished in vitro surfactant activities in comparison with native SP-C and this

155 may be related to their reduced helical content in lipid environments (Johansson et al., 1995). In

156 agreement with these findings, synthetic 34-residue SP-Cff (Fig. 1) showed low solubility in chloroform-

157 methanol, and was difficult to formulate with Tanaka lipids in an active $\alpha$-helical conformation (Walther et

158 al., 2000). A range of in vitro, in vivo and clinical studies have assessed the structural and functional

159 properties of a recombinant, 35-residue SP-Cff (i.e., rSP-C or Venticute ${ }^{\circledR}$ in Fig. 1). Extensive 2D-NMR

160 analyses (Luy et al., 2004; Kairys, Gilson \& Luy, 2004) indicated that freshly prepared recombinant SP-C

161 in chloroform-methanol is a mixture of monomers and dimers, with a $\alpha$-helical conformation (residues

162 Phe-5 to Leu-34) that largely overlaps that of porcine SP-C (Johansson et al., 1994). Non-palmitoylated 
163 rSP-C shares key metastable characteristics of native porcine SP-C when suspended in chloroform-

164 methanol for several days, such as the conversion of $\alpha$-helical conformers to insoluble $\beta$-sheet multimers

165 (Luy et al., 2004). Although early in vivo studies with this recombinant SP-Cff showed surfactant

166 activities closely resembling those of native SP-C (Ikegami \& Jobe, 1998), a recent clinical trial of

167 patients with ARDS demonstrated that rSP-C surfactant neither decreased mortality nor improved patient

168 oxygenation (Spragg et al., 2011). Pulsating bubble surfactometry results suggested that this may have

169 been due to a partial surfactant inactivation during resuspension of rSP-C prior to administration (Spragg

170 et al., 2011). Consequently, the low or unstable surfactant activities observed for the above SP-Css and

171 SP-Cff peptides (Fig. 1) argue that further sequence changes are required to produce more effective, non-

172 palmitoylated SP-C mimics.

173 Accordingly, a second generation of SP-C mimics lacking palmitoyl groups, but with enhanced $\alpha-$

174 helical content and surfactant activities through amino-acid substitutions in the primary sequence, is now

175 being developed for surfactant replacement therapy. The chemically synthesized SP-C33 (Fig. 1) is a

176 second generation SP-C mimic, in which the adjacent palmitoylcysteine groups are replaced with serines

177 and the poly-valines in the $\alpha$-helix are substituted with multiple leucines. In vitro and in vivo studies

178 demonstrated surfactant activities for this SP-C33 analog comparable to those of native SP-C (Johansson

179 et al., 2003; Almlen et al., 2011) and this may be because SP-C33 and native SP-C both similarly insert

180 into surfactant lipids as slightly tilted, transmembrane $\alpha$-helices (Vandenbussche et al., 1992; Pastrana,

181 Maulone \& Mendelsohn, 1991; Almlen et al., 2011). These results support the hypothesis that the

182 manipulation of native sequences may produce SP-C mimics with enhanced $\alpha$-helicity and surfactant

183 activity. However, SP-C33 may not be completely optimized for such important characteristics as

184 surfactant activity (alone or in combination with other surfactant components such as SP-B mimics),

185 resistance to inhibition, toxicity or shelf-life stability. Less invasive second generation SP-C mimics using

186 fewer substitutions may permit more accurate control over critical surfactant properties.

Another strategy for obtaining second generation SP-C mimics without palmitoyl groups involves

188 the introduction of charged ion-pairs (i.e., 'salt-bridges' or 'ion-locks') into SP-C peptides, in which the 
cationic $\mathrm{Lys}^{+}$or $\mathrm{Arg}^{+}$residues interact with anionic Glu${ }^{-}$or Asp ${ }^{-}$residues. In Circular Dichroism (CD) experiments, Marqusee \& Baldwin (1987) showed that incorporation of $\mathrm{Lys}^{+}$and Glu at intervals of 4 residues (i.e., " $i+4$ ") in the sequence substantially increased the helix content of the host peptide. This stabilization of the helix in aqueous environments is due to the formation of an electrostatically neutral ion-pair via the positive- and negative-charged side groups for the $\mathrm{Lys}^{+}$and Glu- residues. Using an augmented Wimley-White (WW) hydrophobicity scale, which accounts for whole-residue energy contributions by the peptide backbone, side-chains and salt-bridge pairs, Membrane Protein Explorer (MPEx) analyses predicted that 'ion-locks' such as $\mathrm{Glu}^{-}-\mathrm{Lys}^{+}$may increase the helicity and lipidpartitioning of peptides and proteins in membranes (Snider et al., 2009). Because the formation of an ionpair is electrostatically neutral, MPEx showed that there is only a modest thermodynamic penalty for replacing the hydrophobic residues of a transmembrane sequence with an 'ion-lock' pairing (e.g., Glu' and Lys $^{+}$) (Jayasinghe, Hristova \& White, 2001). Salt-bridges (or 'ion-locks') have been found in numerous proteins such as $\mathrm{Ca}^{2+}$-ATPase, which has a membrane helix stabilized by an intrahelical charge-pair when 202 facing lipids (Bano-Polo et al., 2012). Importantly, electrostatic calculations indicated that salt-bridges 203 with close-range distances $(\leq 4.0 \AA)$ between the cationic residue "N" and the nearest anionic residue "O" 204 are mostly $\alpha$-helix stabilizing, while those with long-range distances (> $5 \AA$ ) may be helix destabilizing 205 (Kumar \& Nussinov, 1999 \& 2002). and SP-Cff) lacking palmitoyl groups, an SP-C mimic with elevated surfactant activity (SP-C33 UCLA), and SP-C mimics with single or double salt-bridges in the mid-section of the hydrophobic C-terminal region (i.e., SP-Css ion-lock 1, SP-Cff ion-lock 1, SP-C33 ion-lock 1 and SP-C33 ion-lock 2; see Fig. 1).

210 FTIR spectroscopy of SP-Css and SP-Cff in surfactant lipids, an aqueous buffer or a $\alpha$-helix promoting

211 HFIP buffer (Crescenzi et al., 2002; Rajan et al., 1997) indicated elevated $\beta$-sheet, while the corresponding

212 spectra of the daughter SP-Css ion-lock 1 and SP-Cff ion-lock 1 in these environments demonstrated low

$213 \beta$-sheet and mostly high $\alpha$-helix. However, FTIR spectroscopy also indicated that the $\alpha$-helicity of SP-Css

214 ion-lock 1 in surfactant lipids was higher than that of SP-Cff ion-lock 1. Furthermore, oriented and 
215 hydrogen/deuterium (H/D) exchange FTIR spectra showed that an SP-Css ion-lock 1 incorporates into

216 lipid bilayers as a slightly tilted transmembrane $\alpha$-helix, while SP-Cff ion-lock 1 with its shorter helix had

217 a greater tilt and may be restricted to only one monolayer. Consequently, the much higher in vitro and in

218 vivo surfactant activities reported here for SP-Css ion-lock than for SP-Cff ion-lock 1 are attributed to the

219 former SP-C mimic more closely simulating the enhanced $\alpha$-helicity and membrane topography of the

220 native dipalmitoylated SP-C. These results provide a proof of concept that selective insertion of salt-

221 bridge(s) may produce stable and functional SP-C mimics that completely block the well-known

222 amyloidogenic propensities of the parent SP-C protein.

223

224 MATERIALS \& METHODS

225 Ethics Statement

226 The animal study was reviewed and approved by the Institutional Animal Care and Use

227 Committee of the Los Angeles Biomedical Research Institute at Harbor-UCLA Medical Center (protocol \#

228 12958). All procedures and anesthesia were performed in accordance with the American Veterinary

229 Medical Association guidelines.

230

\section{Materials}

232 All organic solvents for sample synthesis were HPLC grade or better. Phospholipids were

233 obtained from Avanti Polar Lipids, Inc. (Alabaster, AL). Deuterated water was NMR quality from Aldrich

234 Chemical Co (St. Louis, MO).

235

236 Synthesis of SP-C Peptides 
238 Css ion-lock 1 and the 33-residue SP-C33 UCLA, SP-C33 ion-lock 1 and SP-C33 ion-lock 2 (Figure 1),

239 was synthesized with FastMoc ${ }^{\mathrm{TM}}$ (Fields et al., 1991) or standard Fmoc procedures on a Leu-HMPB

240 NOVA resin using ABI 431A (Applied Biosystems, Foster City, CA), Symphony Multiple Peptide (Protein

241 Technologies, Tucson, AZ) or Liberty Microwave Peptide (CEM Corp, Matthews, NC) synthesizers. The

242 SP-C mimic peptides were each carboxylated at the C-terminus and amidated at the N-terminus, and all

243 residues were double-coupled to insure optimal yield (Waring et al., 2005). C-terminal carboxyl SP-C

244 peptides were cleaved from the resin using the standard phenol:thioanisole:ethanedithiol:

245 water:trifluoracetic acid (0.75:0.25:0.5:0.5: 10, v:v) cleavage-deprotection mixture (Walther et al., 2010).

246 Crude peptides were then purified (better than 95\%) by preparative HPLC using a VYDAC diphenyl or

247 C8 (1" by 12" width by length) column at $20 \mathrm{ml} / \mathrm{min}$. SP-C peptides were each eluted from the column

248 with a 0 to $100 \%$ (water to acetonitrile with $0.1 \%$ TFA as an ion pairing agent added to both aqueous and

249 organic phases) linear gradient in one hour. The purified product was freeze-dried directly and the mass

250 was confirmed by Maldi TOF mass spectrometry.

\section{Attenuated Total Reflectance-Fourier Transform Infrared (ATR-FTIR) Spectroscopy}

Infrared spectra were recorded at $37^{\circ} \mathrm{C}$ for selected SP-C peptides in Fig. 1 using a Bruker Vector

25422 FTIR spectrometer (Pike Technologies, Madison, WI) with a deuterium triglyceride sulfate (DTGS)

255 detector, averaged over 256 scans at a gain of 4 and a resolution of $2 \mathrm{~cm}^{-1}$ (Waring et al., 2005; Walther et

256 al., 2007b \& 2010; Gordon et al., 2008). For spectral recordings of SP-C peptides in

257 hexafluoroisopropanol (HFIP) or aqueous buffer solutions, self-films were first prepared by air-drying

258 peptide originally in $100 \%$ HFIP onto a $50 \times 20 \times 2 \mathrm{~mm}, 45^{\circ}$ germanium attenuated total reflectance

259 (ATR) crystal for the Bruker spectrometer. The dried peptide self-films were then overlaid with solutions

260 containing 35\% HFIP/65\% deuterated-10 mM sodium phosphate buffer (pD 7.4) (Glasoe \& Long, 1960)

261 or $100 \%$ deuterated $-10 \mathrm{mM}$ sodium phosphate buffer (pD 7.4) before spectral acquisition; control

262 solvent samples were similarly prepared, but without peptide. Spectra of peptides in solvent were obtained 
263 by subtraction of the solvent spectrum from that of the peptide-solvent. For FTIR spectra of SP-C peptides

264 in synthetic surfactant lipid multilayers (DPPC:POPC:POPG; weight ratio, 5:3:2), the peptide was co-

265 solvated with lipid systems in HFIP (lipid:peptide mole ratio of 10:1) and transferred onto an ATR crystal

266 (Pike Technologies). The organic solvent was then removed by flowing nitrogen gas over the sample to

267 produce a lipid-peptide film. The lipid-peptide films were next hydrated ( $\geq 35 \%$ ) (Yamaguchi et al., 2001)

268 with10 mM deuterated sodium phosphate buffer ( $\mathrm{pD} 7.4$ ) vapor in nitrogen for $1 \mathrm{~h}$ prior to acquiring

269 spectra (Gordon et al., 2000; Yamaguchi et al., 2002). The spectra for peptide in lipid mixtures were

270 obtained by subtracting the lipid spectrum hydrated with $10 \mathrm{mM}$ deuterated phosphate buffer (pD 7.4)

271 from that of peptide in lipid with $10 \mathrm{mM}$ phosphate buffer (pD 7.4). Infrared spectra were subsequently

272 recorded for the lipid-peptide films at $37^{\circ} \mathrm{C}$, similar to those described for peptide self-films (see above).

273 Relative amounts of $\alpha$-helix, $\beta$-turn, $\beta$-sheet, or random (disordered) structures in either peptide self-films

274 or lipid-peptide films were estimated using Fourier deconvolution (GRAMS/AI8, version 8.0, Themo

275 Electron Corporation, Waltham, MA) and area of component peaks calculated using curve-fitting software

276 (Igor Pro, version 1.6, Wavemetrics, Lake Oswego, OR) (Kauppinen et al., 1981). FTIR frequency limits

277 were: $\alpha$-helix $\left(1662-1645 \mathrm{~cm}^{-1}\right), \beta$-sheet $\left(1637-1613 \mathrm{~cm}^{-1}\right.$ and $\left.1710-1682 \mathrm{~cm}^{-1}\right)$, turn $/$ bend $\left(1682-1662 \mathrm{~cm}^{-}\right.$

$\left.278^{1}\right)$, and disordered or random (1650-1637 $\left.\mathrm{cm}^{-1}\right)$ (Byler \& Susi, 1986).

279

To estimate the orientation of the $\alpha$-helix of various SP-C peptides in surfactant lipid multilayers

280 deposited on the germanium crystal surface, gold wire polarizers (Perkin Elmer, Waltham, MA) were

281 rotated from $0^{\circ}$ to $90^{\circ}$ to obtain polarized IR spectra of each lipid-peptide film (Gordon et al., 1996). The

282 insertion (or tilt) angle $\Theta$ for the SP-C helical axis with respect to the normal of the germanium surface

283 was calculated based on the dichroic ratio $R$ (Beevers \& Kukol, 2006), which is equal to the ratio of

284 Aparallel to $A_{\text {perpendicular. Here, }}$ Aparallel and $A_{\text {perpendicular }}$ are defined as the respective areas of

285 absorbance at $0^{\circ}$ (parallel) and $90^{\circ}$ (perpendicular) polarizations for the amide I band centered at $\sim 1656$

$286 \mathrm{~cm}^{-1}$. These measures assumed a thick film approximation with the values for the electric field components

287 of the evanescent wave to be $E_{x}=1.398, E_{y}=1.516, E_{z}=1.625$, and an angle $\alpha=39^{\circ}$ for the vibrational

288 dipole relative to the molecular axis of the helix to derive an order parameter $S$ (Beevers \& Kukol, 2006; 
Chia et al., 2002). Here, $S$ is equal to $\left[\left(\mathrm{E}_{x}^{2}-R E_{y}^{2}+E_{z}^{2}\right) /\left(\mathrm{E}_{x}^{2}-R E_{y}^{2}+E_{z}^{2}\right)\right] \div\left[\left(3 \cos ^{2} \alpha-1\right) / 2\right]$. The experimental tilt angle, $\Theta$, was then calculated from this order parameter, by noting that $S=\left(3 \cos ^{2} \Theta-\right.$

1)/2. However, it should be noted that the multilayer peptide-lipid samples stacked on the germanium

this introduces an isotropic contribution that will artificially reduce the dichroic ratio $R$. Accordingly, the lower (Beevers \& Kukol, 2006).

For selected SP-C peptides in surfactant lipids, the stability of the $\alpha$-helical component was also analyzed by monitoring the hydrogen/deuterium (H/D) exchange for amide protons with the FTIR spectrometer. The peptide-lipid sample on the ATR crystal was flushed with $10 \mathrm{mM}$ deuterated phosphate buffer ( $\mathrm{pD} 7.4$ ) vapor in nitrogen at $37^{\circ} \mathrm{C}$, and the decay of the amide II band area $\left(1525-1565 \mathrm{~cm}^{-1}\right)$ was determined as a function of time $(0-6 \mathrm{~h})$ to assess the time-dependent H/D exchange of the amide group (Vandenbussche et al., 1992; Beevers \& Kukol, 2006; Almlen et al., 2011).

\section{Prediction of Aggregation-Forming Regions in SP-C Peptides} aqueous buffer or the lipid-water interface of membranes (Gordon et al., 2008). The PASTA algorithm systematically calculates the relative energies of the various pairing arrangements by assessing a pair-wise energy function for residues facing one another within a $\beta$-sheet. Using a database of known 3D-native structures, PASTA computes two different propensity sets depending on the directionality (i.e., parallel or

311 antiparallel $\beta$-sheets) of the neighboring strands. PASTA assigns relative energies to specific $\beta$-pairings of

312 two sequence stretches of the same length, and assumes that the lower relative energies enhance

313 aggregation by further stabilizing the cross- $\beta$ core. Theoretical PASTA predictions of SP-C and SP-C 
314 mimic aggregation were performed by submitting the primary sequences of these peptides (Fig. 1) to the

315 PASTA Version 1.1 (http://protein.cribi.unipd.it/pasta) website (Trovato, Seno \& Tosatto, 2007). PASTA

316 calculates the relative energies of the most stable pairings (i.e., more negative energies denote more stable

317 conformations), and outputs them as an HTML file (maximum pairings: 100). The PASTA energies for the

318 top-ranked pairings may predict whether the $\beta$-sheet domains of one peptide will be more stable than those

319 of other peptides. The energies for these pairings may also be used to compute an aggregation profile for

320 those regions within a peptide most likely to aggregate as $\beta$-sheets. One cautionary note in using the

321 PASTA algorithm is that it does not correct for the actual environmental polarity of a given protein

322 domain, and also does not explicitly account for salt-bridges forming between charged ion-pairs.

323

324

Hydropathy Predictions of SP-C Peptides Inserting into Lipid Bilayers as Transmembrane Helices

Membrane Protein Explorer (MPEx; Version 3.2.9) is a Java program that analyses hydrophobic

lipid-protein interactions in membranes (http://blanco.biomol.uci.edu/mpex). With the hydropathy analysis

mode (Snider et al., 2009), hydropathy plots are produced using the augmented Wimley-White (WW)

whole-residue hydrophobicity scale that predicts transmembrane helices for protein sequences with high

accuracy (Jayasinghe, Hristova \& White, 2001; Liang et al., 2012). This augmented scale is

330

experimentally based on the partitioning of hydrophobic pentapeptides (Wimley, Creamer \& White, 1996)

331 and salt-bridge pairs (Wimley et al., 1996) into $n$-octanol [i.e., solvent mimic of the hydrocarbon (non-

332 polar) region of the bilayer], and accounts for whole-residue energy contributions due to the peptide

333 backbone, side-chains and salt-bridge pairs (Jayasinghe, Hristova \& White, 2001; Snider et al., 2009).

334 Protein sequences may be submitted to the MPEx program, and the resulting plots are presented as

335 hydropathy $(\mathrm{kcal} / \mathrm{mol})$ versus the sequence residue number, averaged over a sliding window of 19 amino-

336 acid residues. Higher positive hydropathy values reflect enhanced lipid bilayer partitioning for any

337 putative membrane helices. The default scale for the hydropathy analysis uses $n$-octanol, and there is an

338 option for engaging potential salt-bridges using an "on-off” switch on the MPEx control panel. 
In Vitro Adsorption Surface Pressure Measurements

341 Adsorption experiments were done at $37 \pm 0.5^{\circ} \mathrm{C}$ in a Teflon ${ }^{\circledR}$ dish with a $35 \mathrm{ml}$ subphase $(0.15 \mathrm{M}$

$342 \mathrm{NaCl}+1.5 \mathrm{mM} \mathrm{CaCl}_{2}$ ) stirred to minimize diffusion resistance as described previously (Notter,

343 Finkelstein \& Taubold, 1983). Surfactants were prepared by co-solvating the lipid components (i.e.,

344 DPPC:POPC:POPG, 5:3:2, weight ratio) with the SP-C peptide in organic solvent HFIP (100\%), dried

345 under nitrogen, exposed to house vacuum to remove residual solvent, resuspended by hand vortexing in

$3460.15 \mathrm{M} \mathrm{NaCl}$ (normal saline) adjusted to $\mathrm{pH} 7.0$ with $0.1 \mathrm{~N}$ sodium bicarbonate, heated to $65^{\circ} \mathrm{C}$

347 intermittently for $30 \mathrm{~min}$, and refrigerated for $12 \mathrm{~h}$ prior to use. At time zero, a bolus of these surfactant

348 preparations containing $2.5 \mathrm{mg}$ lipid in $5 \mathrm{ml}$ of $0.15 \mathrm{M} \mathrm{NaCl}+1.5 \mathrm{mM} \mathrm{CaCl}_{2}$ was injected into the stirred

349 subphase, and adsorption surface pressure (surface tension lowering below that of the pure subphase) was

350 measured as a function of time by the force on a partially submerged, sandblasted platinum Wilhelmy

351 slide (Notter, Finkelstein \& Taubold, 1983). The final surfactant concentration for adsorption studies was

352 uniform at $0.0625 \mathrm{mg}$ phospholipid/ml (2.5 $\mathrm{mg}$ surfactant phospholipid/40 $\mathrm{ml}$ of final subphase).

353

354 In Vitro Surfactant Activity Measured with Captive Bubble Surfactometry

355 Adsorption and dynamic surface tension lowering ability of all above described surfactant

356 preparations were measured with a captive bubble surfactometer at physiological cycling rate, area

357 compression, temperature, and humidity (Walther et al., 2010). We routinely analyze surfactant samples of

$3581 \mu \mathrm{l}(35 \mathrm{mg}$ phospholipids/ $\mathrm{ml})$ in the captive bubble surfactometer and perform all measurements in

359 quadruplicate.

360

361

Ventilated, Lung-Lavaged, Surfactant-Deficient Rabbit Model

362

Young adult New Zealand white rabbits (weight 1.0-1.3 kg) received anesthesia with $50 \mathrm{mg} / \mathrm{kg}$ of

363 ketamine and $5 \mathrm{mg} / \mathrm{kg}$ of acepromazine intramuscularly prior to placement of a venous line via a marginal

364 ear vein. After intravenous administration of $1 \mathrm{mg} / \mathrm{kg}$ of diazepam and $0.2 \mathrm{mg} / \mathrm{kg}$ of propofol, a small

365 incision was made in the skin of the anterior neck for placement of an endotracheal tube and a carotid

366 arterial line. After placement of the endotracheal tube muscle paralysis was induced with intravenous 
pancuronium $(0.1 \mathrm{mg} / \mathrm{kg})$. During the ensuing duration of mechanical ventilation anesthesia was

maintained by continuous intravenous administration of $3 \mathrm{mg} / \mathrm{kg} / \mathrm{h}$ of propofol and intravenous dosages of $1 \mathrm{mg} / \mathrm{kg}$ of diazepam as needed; muscle paralysis was maintained by hourly intravenous administration of $0.1 \mathrm{mg} / \mathrm{kg}$ of pancuronium. Heart rate, arterial blood pressures and rectal temperature were monitored continuously. Respiratory function was followed during mechanical ventilation by measurements of serial arterial blood gases and pulmonary compliance over the first 2 hours after a standardized saline lavage protocol. For this timeframe of study, the model reflected a relatively pure state of surfactant insufficiency in animals with mature lungs.

After stabilization on the ventilator, saline lavage was performed with repeated intratracheal instillation and removal of $30 \mathrm{~mL}$ of normal saline until the $\mathrm{PaO}_{2}$ dropped below $100 \mathrm{~mm} \mathrm{Hg}$ (average 3 lavages). Maintenance fluid was provided by a continuous infusion of Lactated Ringer's solution at a rate of $10 \mathrm{ml} / \mathrm{kg} / \mathrm{h}$. Edema fluid appearing in the trachea was removed by suctioning. When the $\mathrm{PaO}_{2} \mathrm{was}$ stable at less than $100 \mathrm{~mm} \mathrm{Hg}$ in saline lavaged animals, an experimental or control surfactant mixture was instilled into the trachea at a dose of $100 \mathrm{mg} / \mathrm{kg}$ body weight and a concentration of $35 \mathrm{mg} / \mathrm{ml}$. The rabbits were ventilated using a Harvard volume controlled animal ventilator (tidal volume $7.5 \mathrm{ml} / \mathrm{kg}$, a

382 positive end-expiratory pressure of $3 \mathrm{~cm} \mathrm{H}_{2} \mathrm{O}$, an inspiratory/expiratory ratio of $1: 2,100 \%$ oxygen, and a

383 respiratory rate to maintain a $\mathrm{PaCO}_{2}$ at $\sim 40 \mathrm{~mm} \mathrm{Hg}$ ). Airway flow and pressures and tidal volume were

384 monitored continuously with a pneumotachograph connected to the endotracheal tube and a pneumotach

385 system. Arterial $\mathrm{pH}$ and blood gases were done every 15 minutes. Dynamic lung compliance was

386 calculated by dividing tidal volume/kg body weight by changes in airway pressure (peak inspiratory

387 pressure minus positive end-expiratory pressure $)\left(\mathrm{ml} / \mathrm{kg} / \mathrm{cm} \mathrm{H}_{2} \mathrm{O}\right)$. Animals were sacrificed 2 hours after

388 surfactant administration with an overdose of pentobarbital. End-points were pulmonary gas exchange

389 (arterial $\mathrm{pH}, \mathrm{PaCO}_{2}$ and $\mathrm{PaO}_{2}$ ) and pulmonary mechanics (dynamic compliance).

\section{RESULTS}




\section{Ion-Locks}

To test the structural effects of inserting ion-locks into SP-C peptides, a suite of SP-C mimics was

395 prepared based on native SP-C sequences (i.e., SP-Css and SP-Cff) and these native SP-C sequences with

396 a Glu-Lys $^{+}$pairing in the hydrophobic midsection (i.e., SP-Css ion-lock 1 and SP-Cff ion-lock 1; see Fig.

397 1). Representative FTIR spectra of the amide I band for SP-Css and SP-Cff in surfactant lipids

398 (DPPC:POPC:POPG 5:3:2, weight ratio) in Fig. 2A were similar, each showing a major $\beta$-sheet

399 component with a predominant peak at $\sim 1630-1631 \mathrm{~cm}^{-1}$ and a miniscule companion peak at $\sim 1691 \mathrm{~cm}^{-1}$,

400 and a lower $\alpha$-helical component with a mid-field shoulder at $\sim 1657 \mathrm{~cm}^{-1}$. Subsequent deconvolutions

401 confirmed that SP-Css and SP-Cff in surfactant lipids principally adopt $\beta$-sheet conformations ( $\geq \sim 58 \%)$

402 with minor contributions from $\alpha$-helix, loop-turn and disordered structures (Table 1). In additional studies

403 (Table 2), FTIR spectra and deconvolutions for SP-Css and SP-Cff in a membrane-interfacial lipid mimic

404 (i.e., 35\% HFIP/65\% deuterated-sodium phosphate buffer, pD 7.4) or 100\% aqueous buffer (i.e., $10 \mathrm{mM}$

405 deuterated phosphate buffer, pD 7.4) indicated comparably high $\beta$-sheet ( $\geq 36-50 \%)$, with smaller

406 proportions from other conformations. Consistent with these findings, prior FTIR spectroscopy of 34-

407 residue SP-Cff showed reduced $\alpha$-helix on short-term storage in surfactant lipids (Walther et al. 2000),

408 while earlier CD analyses of synthetic, 35-residue SP-Css or SP-Cff in detergent micelles also indicated

409 enhanced $\beta$-sheet and reduced $\alpha$-helix $(<20 \%)$ (Johansson et al., 1995). In our hands, SP-Css and SP-Cff

410 exhibited only high $\beta$-sheet and low $\alpha$-helix in surfactant lipids (Fig. 2; Table 1) or environments of

411 differing polarity (Table 2).

412 FTIR spectroscopy determinations for either SP-Css ion-lock 1 or SP-Cff ion-lock 1 in surfactant

413 lipids indicated enhanced $\alpha$-helix and reduced $\beta$-sheet, when compared to the corresponding

414 measurements of the native SP-Css or SP-Cff sequences. For example, FTIR spectra of SP-Css ion-lock 1

415 in surfactant lipids (Fig. 2A) indicated high $\alpha$-helix (at $\sim 1657 \mathrm{~cm}^{-1}$ ), and very low $\beta$-sheet (at $\sim 1630 \mathrm{~cm}^{-1}$ ).

416 Importantly, deconvolution showed predominant $\alpha$-helix (i.e., $\sim 63 \%$ ) and minor $\beta$-sheet ( $\sim 6 \%$ ) for SP-Css

417 ion-lock 1 in surfactant lipids, as opposed to the reduced $\alpha$-helix $(\sim 25 \%)$ and elevated $\beta$-sheet $(\sim 58 \%)$ 
determined from FTIR spectra of its parent SP-Css in this environment (Fig. 2A; Table 1). Consequently, insertion of the ion-lock (i.e., Glu--20-Lys $\left.{ }^{+}-24\right)$ in the mid-section of the poly-Val sequence produced a remarkable conformation shift in the host SP-Css, from an 'amyloid-like' $\beta$-sheet profile to one that is

421 primarily $\alpha$-helix (Fig. 2A). Additional FTIR spectra of SP-Css ion-lock 1 in aqueous and membrane422 interfacial HFIP milieu also demonstrated elevated $\alpha$-helix values ( $\geq \sim 41 \%)$ over those obtained for the 423 parent SP-Css (Table 2), suggesting that an intrapeptide salt-bridge (at Glu-20-Lys ${ }^{+}-24$ ) stabilizes the $\alpha$ 424 helical conformation in environments of differing polarity. In further studies, FTIR spectra of SP-Cff ion425 lock 1 in surfactant lipids showed enhanced $\alpha$-helix (at $\sim 1653 \mathrm{~cm}^{-1}$ ) and low $\beta$-sheet $\left(\right.$ at $\sim 1630 \mathrm{~cm}^{-1}$ ) (Fig. 426 2A). Deconvolution of these SP-Cff ion-lock 1 spectra verified that the major structural component in 427 lipids was $\alpha$-helix ( $44.6 \%$ ), produced by a conformational change from the very high $\beta$-sheet $(\sim 74.3 \%)$ 428 of its parent SP-Cff (Fig. 2A; Table 1). Incorporation of the ion-pair (i.e., Glu-20-Lys ${ }^{+}-24$ ) induced a 429 similar conformational shift for SP-Cff in the lipid-mimic HFIP solution from a $\beta$-sheet conformation $430(\sim 53.2 \%)$ to one that is predominately $\alpha$-helix $(\sim 53.0 \%)$ (Table 2$)$. Although these FTIR spectral results 431 suggest at least a partial salt-bridge for SP-Cff ion-lock 1 in lipid and lipid-mimic environments, 432 additional FTIR experiments indicate that this salt-bridge may not be as stable as that seen with SP-Css 433 ion-lock 1. Unlike SP-Css ion-lock 1, SP-Cff ion-lock 1 folds primarily as loop-turns in aqueous buffer

434 (Table 2) and even shows diminished $\alpha$-helix content in surfactant lipids relative to that of SP-Css ion-lock 4351 (i.e., $\sim 44.6$ vs. $\sim 63.1 \%$ in Fig. $2 \mathrm{~A}$ and Table 1 ). Such $\alpha$-helical reductions may be partially due to the Phe 436 groups of SP-Cff ion-lock 1 (Fig. 1) weakening the putative salt-bridge between Glu-20-Lys $^{+}-24$, either 437 through direct or indirect mechanisms (see below).

It is also worthwhile to contrast the helical properties determined for FTIR spectra of SP-Css ion439 lock 1 and SP-Cff ion-lock 1 with those of SP-C33 UCLA and several SP-C33 ion-locks. SP-C33 UCLA 440 has the same sequence as SP-C33 (Almlen et al., 2010 \& 2011) with poly-Leu substituting for the poly441 Val in the C-terminal region (Fig. 1). Previous CD and FTIR studies have reported that SP-C mimics of 442 the poly-Leu class, including SP-C33 (Almlen et al., 2011) and its homologous precursor SP-C(Leu) 443 (Nilsson et al., 1998), each show elevated $\alpha$-helix when incorporated into lipids or lipid-mimics. In 
444 support of these experimental results, earlier Molecular Dynamics simulations of SP-C33 in methanol for

$44510 \mathrm{nsec}$ indicated that the hydrophobic poly-Leu region adopted an $\alpha$-helical conformation in this

446 membrane-interfacial mimic (Almlen et al., 2010). The FTIR spectrum of SP-C33 UCLA in surfactant

447 lipids overlapped that of SP-Css ion-lock 1 (Fig. 2A and B), and spectral deconvolution indicated

448 similarly high $\alpha$-helix (Table 1). Moreover, FTIR spectra of SP-C33 UCLA in either aqueous or HFIP

449 solutions showed $\alpha$-helical components (at $\sim 1653-1655 \mathrm{~cm}^{-1}$ ) nearly identical to those of SP-Css ion-lock

4501 (not shown), as well as similar $\alpha$-helix levels (i.e., $440-51 \%$ ) using spectral deconvolution (Table 2).

451 Although SP-Css ion-lock 1 and SP-C33 UCLA indicate comparably high $\alpha$-helix in aqueous buffer,

452 aqueous HFIP buffer or surfactant lipids (Fig. 2: Tables 1 and 2), SP-C33 UCLA requires replacement of

453 the poly-Val sequence with ten Leu residues to achieve this stabilization. Contrarily, SP-Css ion-lock 1

454 needs only a single ion-pair at Glu-20-Lys ${ }^{+}-24$ to maintain high $\alpha$-helix. In further research, the SP-C33

455 ion-lock 1 and SP-C33 ion-lock 2 mimics were prepared by inserting a single (i.e., Glu-20-Lys $\left.{ }^{+}-24\right)$ or

456 double (i.e., $\mathrm{Lys}^{+}-15-\mathrm{Glu}^{-}-19$ and Glu-20-Lys ${ }^{+}-24$ ) ion-lock into the SP-C33 sequence (Fig. 1). FTIR

457 spectra and deconvolution indicated $\alpha$-helix for these SP-C33 ion-locks in aqueous, HFIP-water and

458 surfactant lipids similar to those of SP-C33 (Fig. 2; Tables 1 and 2). For example, the FTIR spectra of SP-

459 C33 UCLA and the SP-C33 ion-locks in surfactant lipids nearly overlap with $\alpha$-helix maxima at $1655 \mathrm{~cm}^{-1}$

460 (Fig. 2B), and spectral deconvolutions of each show elevated $\alpha$-helix of $\sim 65-67 \%$ (Table 1). Because the

461 insertions of either one or two charged ion-pairs were unable to raise the $\alpha$-helical content beyond that of

462 the parent SP-C33 UCLA (Tables 1 and 2), the SP-C33 primary sequence may already be maximized for

$463 \alpha$-helix. Interestingly, the $\alpha$-helicity of SP-Css ion-lock 1 in surfactant lipids (i.e., $\sim 63 \%$ ) is only slightly

464 below those of the SP-C33 and the SP-C33 ion-locks (i.e., 65-67\%), suggesting that the SP-Css ion-lock

4651 in lipids is also almost optimized for $\alpha$-helix.

466

467

Effects of Ion-Locks on the Aggregation Domains of SP-C Peptides Detected with PASTA Analysis

PASTA calculations were next performed to assess whether the enhanced $\alpha$-helicity induced by ion-lock insertion into either SP-Css or SP-Cff (Fig. 2; Tables 1 and 2) is related to the disruption of $\beta$ - 
sheet regions in the host peptides. The PASTA algorithm systematically determines the relative energies

471 for various paired sequences by assessing an energy function for facing residues that are H-bonded within

472 a $\beta$-sheet (Trovato et al., 2006). Here, PASTA predicted that the native SP-Cff and SP-Css peptides will

473 each form $\beta$-sheet at their respective C-terminal domains. Specifically, PASTA energy calculations showed

474 that the most likely pairing for SP-Cff or SP-Css will be an interpeptide, parallel $\beta$-sheet [i.e., parallel in-

475 register arrangement (PIRA)] for the C-terminal residues 7-27 (Fig. 1) with a relative PASTA energy of

476 -29.2 (Trovato et al., 2006; Trovato, Seno \& Tosatto, 2007). Using a database of 179 peptides as a

477 benchmark (Trovato, Seno \& Tosatto, 2007), an energy threshold of $<-29.0$ indicates a probability $>98 \%$

478 (true positive rate) that SP-Cff and SP-Css are each "amyloid-like" (i.e., have a high $\beta$-sheet tendency) for

479 the corresponding C-terminal segments (i.e., residues 7-27). This prediction of elevated $\beta$-sheet for SP-Css

480 and SP-Cff was confirmed by FTIR spectra of these peptides in surfactant lipids with high $\beta$-sheet and low

$481 \alpha$-helix (Figs. 2 and 3; Table 1). Furthermore, Figure 3 shows that incorporation of Glu-20-Lys ${ }^{+}-24$ in

482 either SP-Cff or SP-Css increased the relative PASTA energy from -29.2 to -22.2 for the 7-27 sequence,

483 and also raised the $\alpha$-helix levels of the particular SP-C ion-locks in surfactant lipids. Because FTIR

484 deconvolutions (Fig. 2; Table 1) demonstrated that the enhanced $\alpha$-helix in SP-Css ion-lock 1 or SP-Cff

485 ion-lock 1 in lipids occurred concomitantly with reduced $\beta$-sheet, the charged ion-pair may partially

486 augment $\alpha$-helicity by disrupting $\beta$-sheet in the $\mathrm{C}$-terminal region.

Interestingly, Figure 3 shows that an analogous insertion of ion-locks in SP-C33 does not increase the $\alpha$-helix levels of SP-C33 ion-lock 1 and SP-C33 ion-lock 2 in surfactant lipids, despite the relative

489 PASTA energies for these daughter ion-locks being substantially higher than that of the parent SP-C33.

490 The inability of ion-locks to further raise the $\alpha$-helicity of SP-C33, unlike the large $\alpha$-helix increases seen

491 for ion-lock incorporation into SP-Css and SP-Cff, may be due to the already high $\alpha$-helix and low $\beta$-sheet

492 present in the SP-C33 peptide (Fig. 3). FTIR spectral deconvolutions for SP-C33, SP-C33 ion-lock 1 and

493 SP-C33 ion-lock 2 in surfactant lipids each indicated $<\sim 8 \% \beta$-sheet (Table 1), and additional recruitment

494 of $\alpha$-helix from $\beta$-sheet residues may not be possible. In this context, SP-Css ion-lock 1 in surfactant lipids 
495

496

497

498

499

500

501

502

503

504

505

506

507

508

509

510

511

512

513

514

515

516

517

518

519

may be similarly optimized for high $\alpha$-helix, as FTIR deconvolutions showed elevated $\alpha$-helix and diminished $\beta$-sheet matching those of SP-C33 and SP-C33 ion-locks (Table 1).

\section{Prediction of Transmembrane $\alpha$-Helices for SP-C Mimics using MPEx Hydropathy Calculations}

Because secondary structure analysis using FTIR spectroscopy or PASTA do not directly measure membrane binding of helical peptides, the tendency of SP-C peptides to form transmembrane $\alpha$-helices was next evaluated with hydropathy calculations using the Membrane Protein Explorer (MPEx) program. MPEx determines the hydropathy $(\mathrm{kcal} / \mathrm{mol})$ for protein sequences using the augmented Wimley-White (WW) hydrophobicity scale and is based on measurements of the partitioning of hydrophobic pentapeptides and salt-bridge pairs into $n$-octanol (Jayasinghe, Hristova \& White, 2001). Previous MPEx calculations have predicted transmembrane helices for protein sequences with high accuracy and are especially useful in preliminary characterizations of novel proteins or peptides (Snider et al., 2009; Liang et al., 2012). One important feature of MPEx is its option for engaging potential salt-bridges using an "enter-remove" icon on the control panel (Snider et al., 2009). However, note that dipalmitoylated SP-C is excluded from the below comparisons, because the MPEx and PASTA algorithms do not correct for how covalently attached, non-peptide moieties (e.g., the palmitoyl groups in native SP-C; Fig. 1) may modify membrane incorporation or peptide aggregation, or both.

As a surrogate for native SP-C, the rSP-C sequence was submitted to MPEx, which predicted that this SP-C mimic would insert into lipid bilayers as a transmembrane $\alpha$-helix with its most favorable hydropathy (i.e., $11.3 \mathrm{kcal} / \mathrm{mol}$ ) through the C-terminal segment 12-30. The rSP-C sequence was chosen as a control for our MPEx analyses of SP-C ion-locks, because the recombinant protein is highly homologous with the dipalmitoylated SP-C (Fig. 1), lacks the two palmitoylcysteine groups of the native protein and has been previously subjected to structural and functional experiments. To simultaneously visualize the respective propensities of SP-C peptides to insert into lipids as transmembrane helix and to form $\beta$-sheet, the "Hydropathy from MPEx" (ordinate) is plotted versus the "Relative PASTA Energy" 
520 (abscissa) for various SP-C mimics (Figure 4). The more positive hydropathy values (i.e., bottom-to-top)

521 reflect greater incorporation as a transmembrane helix, while higher relative PASTA energy values (i.e.,

522 left-to-right) represent a lower tendency to form $\beta$-sheet. If rSP-C binds to lipid bilayers with the same

523 helical conformation as in chloroform-methanol (i.e., $\alpha$-helix for residues 9-34) (Luy et al., 2004), then

524 Fig. 4 predicts that rSP-C readily incorporates as a transmembrane $\alpha$-helix through its C-terminus. The

525 very low PASTA energy indicated for rSP-C (Fig. 4) may also be responsible for the metastable helical

526 properties observed in earlier 2D-NMR studies (Luy et al., 2004), as $\beta$-sheet multimers or fibrils should

527 here be thermodynamically favored over $\alpha$-helix (Fig. 3) (Trovato et al., 2006; Trovato, Seno \& Tosatto, 528 2007).

529

The relative tendencies of SP-Css ion-lock 1 and SP-Cff ion-lock 1 to penetrate into lipid bilayers

530 as transmembrane $\alpha$-helices were also predicted with the joint MPEx and PASTA plot in Fig. 4. Earlier

531 MPEx research showed that hydrophobic helices bearing salt-bridges may penetrate deeply into lipid

532 bilayers, because there is only a slight thermodynamic penalty for replacing the hydrophobic residues of a

533 transmembrane sequence with a neutral, charged ion-pair (e.g., Glu${ }^{-}$and $\mathrm{Lys}^{+}$) (Snider et al. 2009;

534 Jayasinghe, Hristova \& White, 2001). Moreover, previous electrostatic calculations indicated that salt-

535 bridges with close-range distances $(\leq 4.0 \AA)$ between the cationic residue "N" and the nearest anionic

536 residue "O" are mostly $\alpha$-helix stabilizing, while disengaged salt-bridges with long-range distances $(>5$

$537 \AA$ ) may be helix destabilizing (Kumar \& Nussinov, 1999 \& 2002). As indicated above, MPEx allows

538 hydropathic analysis of SP-C ion-lock sequences in membranes assuming the salt-bridge is either engaged

539 (i.e., close-range salt-bridge) or disengaged (i.e., long-range salt-bridge). With a close-range salt-bridge

540 between the Glu-20-Lys ${ }^{+}-24$ pair, MPEx forecasts that a highly $\alpha$-helical SP-Css ion-lock 1 would deeply

541 embed into the lipid bilayer due to the high hydropathy (i.e., $\sim 10.3 \mathrm{kcal} / \mathrm{mol}$ ) of its segment 12-30.

542 Alternatively, with a long-range salt-bridge between Glu-20-Lys ${ }^{+}-24$, the corresponding MPEx

543 hydropathy would be much lower (i.e., $\sim 4.0 \mathrm{kcal} / \mathrm{mol}$ ) and predicts a more superficial association between

544 a less helical SP-Css ion-lock 1 and the bilayer. Our FTIR results indicating high $\alpha$-helicity for SP-Css

545 ion-lock 1 in surfactant lipids are consistent with a close-range salt-bridge forming in this environment, 
permitting deep insertion of this SP-C mimic as a transmembrane $\alpha$-helix. Specifically, FTIR deconvolution in Table 1 showed that an SP-Css ion-lock 1 in surfactant lipids is highly $\alpha$-helical ( $\sim 63.1 \%)$. Thus, the SP-Css ion-lock $1 \alpha$-helix ( $\sim 21$ residues) may span the width of the bilayer, as it lies within the 17-25 residue window reported for other transmembrane $\alpha$-helices (Liang et al., 2012). The PASTA prediction that the Glu-20-Lys ${ }^{+}-24$ pair in SP-Css ion-lock 1 converts segment 7-27 from $\beta$-sheet to $\alpha$-helix (Figs. 3 and 4) also supports the MPEx assignment that $\alpha$-helix is confined to the C-terminal region. Consequently, helical SP-Css ion-lock 1 with a close-range salt-bridge shows a high hydropathy, which is only slightly less than that of rSP-C (i.e., 10.3 vs. $11.3 \mathrm{kcal} / \mathrm{mol}$ in Fig. 4), and should mimic the transmembrane incorporation of native SP-C peptides. On the other hand, the reduced $\alpha$-helicity observed for SP-Cff ion-lock 1 in surfactant lipids is compatible with a disruptive, long-range salt-bridge that lowers $\alpha$-helical levels to $\sim 44.6 \%$ (Table 1 ). The resulting SP-Cff ion-lock $1 \alpha$-helix is too short ( $\sim 15$ residues) to span the width of the bilayer (Liang et al., 2012) and instead may be limited to a single monolayer. These studies suggest that the replacement of the two serines in SP-Css ion-lock 1 with vicinal phenylalanines disorders the close-range, salt-bridge between $\mathrm{Glu}^{-}-20-\mathrm{Lys}^{+}-24$, through either direct or 560 indirect mechanisms.

FTIR secondary structure and theoretical analyses similarly examined the insertion of SP-C33

562 mimics into the lipid bilayer as transmembrane $\alpha$-helices. Fig. 4 indicates higher MPEx and PASTA 563 energy values for SP-C33 relative to those of rSP-C, predicting that the swap of poly-Leu with the poly-

564 Val sequence of native SP-C produces an SP-C33 mimic that associates with lipids as a transmembrane $\alpha-$ 565 helix. In support of this assignment, FTIR showed that the principal spectral component for SP-C33

566 UCLA in surfactant lipids was $\alpha$-helix and that the $\alpha$-helicity (67.2\% from Table 1$)$ for SP-C33 UCLA is

567 sufficiently high for this SP-C mimic to incorporate as a transmembrane helix ( $\sim 22$ residues long). In this

568 context, Fig. 4 also predicts that SP-C33 ion-lock 1 or SP-C33 ion-lock 2 (i.e., SP-C33 mimics with one or

569 two Glu-20-Lys ${ }^{+}-24$ pairs; Fig. 1) will incorporate into lipid bilayers either as transmembrane $\alpha$-helices

570 with close-range salt-bridges or disordered $\alpha$-helices with long-range salt-bridges and reduced membrane

571 affinity. Because our FTIR results show only high $\alpha$-helix levels for these SP-C33 ion-locks matching 
572 those of the parent SP-C33 in surfactant lipids (Table 1), both SP-C33 ion-lock 1 and SP-C33 ion-lock 2

573 will likely form engaged salt-bridges, and incorporate into surfactant lipids as transmembrane $\alpha$-helices.

\section{Orientation-dependent FTIR Analysis of the Helical Axes of SP-C Mimics in Surfactant Lipids}

576

577

578

579

580

581

582

583

584

585

586

587

588

589

590

591

592

593

594

595

596

597
The topography of helical SP-C mimics in surfactant lipid multilayers was experimentally assessed by recording polarized infrared spectra of each lipid-peptide film that was rotated from $0^{\circ}$ to $90^{\circ}$. Orientation-dependent FTIR spectroscopy of native SP-C (Vandenbussche et al., 1992; Pastrana, Maulone \& Mendelsohn, 1991) and unrelated membrane proteins (Chia et al., 2002; Beevers \& Kukol, 2006) was previously used to determine the $\Theta$ angles between their respective helical axes and the normal to the lipid bilayer plane. Early polarized FTIR studies indicated that native bovine and porcine SP-C proteins (Vandenbussche et al., 1992; Pastrana, Maulone \& Mendelsohn, 1991) each insert into surfactant lipids as a transmembrane helix whose long molecular axis parallels the phospholipid acyl chains and a maximum angle of $\sim 20-24^{\circ}$ with respect to the bilayer normal. In the present research, the polarized FTIR spectra of the amide I band for SP-Css ion-lock 1 in surfactant multilayers were highly anisotropic, with high and low absorbance at $0^{\circ}$ and $90^{\circ}$ (Fig. 5A). The dichroic ratio R for SP-Css ion-lock 1 is computed as the ratio of the integrated amide I absorption at parallel and perpendicular with polarized infrared light. The use of this dichroic $\mathrm{R}$ indicated a maximum tilt angle $\Theta=22.6^{\circ}$ for the helical axis of SP-Css ion-lock 1 with respect to the bilayer normal (Table 1), similar to those determined previously for native SP-C (Vandenbussche et al., 1992; Pastrana, Maulone \& Mendelsohn, 1991). Based on its high $\alpha$-helicity (63.1\%) (Fig. 2A; Table 1), the SP-Css ion-lock 1 probably incorporates in surfactant lipids as a transmembrane $\alpha$-helix ( $\sim 21$ residues long) spanning the bilayer with a maximum $22.6^{\circ}$ tilt, particularly if this SP-C mimic is highly hydropathic due to a salt-bridge between Glu-20 - Lys ${ }^{+}-24$ (Fig. 4). When compared with FTIR results from SP-Css ion-lock 1 or native SP-C, however, the polarized FTIR spectra for SP-Cff ion-lock 1 in surfactant lipids were less anisotropic with a lower dichroic R and demonstrated a correspondingly higher maximum tilt angle $\left(\Theta=34.2^{\circ}\right)($ Table 1$)$. Because of its reduced $\alpha$-helix levels ( 44.6\%) calculated from FTIR spectral deconvolutions and higher maximum tilt angle (Figs. 2A and 5B; 
598 Table 1$)$, the relatively short ( $\sim 15$ residues) $\alpha$-helix of SP-Cff ion-lock 1 in surfactant lipids is probably

599 limited to one bilayer leaflet. One hypothesis worth exploring is that the low $\alpha$-helicity and more oblique

$600 \Theta$ observed for SP-Cff ion-lock is due to a failure of its $\mathrm{Glu}^{-}-20-\mathrm{Lys}^{+}-24$ to form an engaged salt-bridge

601 (i.e., close-range) in surfactant lipids.

602 Analogous oriented FTIR experiments were performed to characterize the membrane topography 603 of SP-C33 and SP-C33 ion-locks in surfactant lipids. As noted above, conventional FTIR analysis of SP-

604 C33 UCLA, SP-C33 ion-lock 1 or SP-C33 ion-lock 2 (Fig. 1) in surfactant lipids each showed spectra 605 with a predominant $\alpha$-helix band centered at $1655 \mathrm{~cm}^{-1}$ (Fig. 2B) and elevated levels (65-67\%) of $\alpha$-helix

606 (Table 1). The polarized FTIR spectra of the amide I bands for SP-C33 UCLA (Fig. 5D), SP-C33 ion-lock

6071 (Fig. 5C) and SP-C33 ion-lock 2 (not shown) were all highly anisotropic, indicating maximum $\Theta$ angles

608 of $\sim 23^{\circ}$ (Table 1). Consequently, SP-C33 UCLA and the SP-C33 ion-locks incorporate into surfactant lipid

609 as transmembrane $\alpha$-helices ( $\sim 21-22$ residues long) spanning the bilayer with a maximum tilt angle of

$610 \sim 23^{0}$, confirming that they accurately reproduce the membrane topography of native SP-C proteins

611 (Vandenbussche et al., 1992; Pastrana, Maulone \& Mendelsohn, 1991). Moreover, SP-C33 ion-lock 1 and

612 SP-C33 ion-lock 2 each probably contain stable salt-bridges as predicted from MPEx-hydropathy analysis

613 (Fig. 4; top right quadrant), which would facilitate the deep penetration of these SP-C mimics into

614 surfactant bilayers. Importantly, our orientation-dependent FTIR results with SP-C33 UCLA (Fig. 5D;

615 Table 1) are in good agreement with those earlier reported for SP-C33 incorporated into surfactant lipid 616 bilayers [46], despite these studies using different experimental conditions for peptide synthesis and FTIR 617 spectral measurements.

618

619 FTIR Analysis of Hydrogen/Deuterium (H/D) Exchange for Amide Protons of SP-C Mimics in 620 Surfactant Lipids

621 For selected SP-C mimics with surfactant lipids, the extent of peptide helix insertion into the lipid 622 bilayer was characterized by measuring the hydrogen/deuterium (H/D) exchange of amide protons. 
623 Previous studies of native SP-C (Vandenbussche et al., 1992; Pastrana, Maulone \& Mendelsohn, 1991)

624 and unrelated membrane proteins (Chia et al., 2002; Grimard et al., 2001) indicated that protons

625 participating in the H-bonds of highly ordered structures such as the transmembrane $\alpha$-helix may show a

626 lack of deuterium exchange for up to 6-28 h. The H/D exchange for amide protons that maintain the

627 transmembrane $\alpha$-helix was assessed as follows. SP-C mimic-lipid samples on the ATR crystal were

628 hydrated by bubbling nitrogen through a $10 \mathrm{mM}$ deuterated phosphate buffer (pD 7.4) at $37^{\circ} \mathrm{C}$, and the

629 amide I and amide II peaks were recorded with FTIR spectra over the range $1500-1700 \mathrm{~cm}^{-1}$ as a function

630 of time $(0-6 \mathrm{~h})$. The peak between $1600-1700 \mathrm{~cm}^{-1}$ reflects the amide I band [i.e., $v(\mathrm{C}=\mathrm{O})$ of the peptide

631 bond] that is sensitive to the secondary conformations of the protein, while the second band between

$6321525-1565 \mathrm{~cm}^{-1}$ is primarily due to amide II [i.e., $\delta(\mathrm{N}-\mathrm{H})$ of the peptide bond] (Vandenbussche et al.,

633 1992; Jackson \& Mantsch, 1995). The ratio of the amide II to amide I peak areas is proportional to the

$634 \mathrm{H} / \mathrm{D}$ exchange, as such exchange would shift the amide II band (at $\sim 1543 \mathrm{~cm}^{-1}$ ) to that of amide II' (at

$635 \sim 1450 \mathrm{~cm}^{-1}$ ) (Pastrana, Maulone \& Mendelsohn, 1991. Contrarily, the absence of H/D exchange would

636 suggest that the H-bonds are isolated from aqueous media (e.g., transmembrane $\alpha$-helices), or are in

637 aqueous environments in ordered conformations where exchange is slow.

638 In Figure 6 the percentage (\%) unexchanged hydrogens for the SP-Css ion-lock 1 in surfactant

639 lipids is shown as a function of time (0-6). Analogous H/D curves for membrane proteins were previously

640 analyzed as the sum of multiple linear exponential (first-order) decays (Grimard et al., 2001). Besides fast-

641 and intermediate- H/D exchange compartments (i.e., with $t_{1 / 2}$ 's between 10 and 120 min involving $49 \%$

642 of the helical residues), there is a final compartment for helical amide hydrogens $(\sim 51 \%)$ that resists H/D

643 exchange for $\mathrm{t} \leq 6 \mathrm{~h}$ (Fig. 6). The inaccessible fraction at long-times is probably due to segments buried

644 deeply as transmembrane $\alpha$-helix, as the residual amide II band remains centered at $\sim 1543 \mathrm{~cm}^{-1}$ (not

645 shown). Because spectral deconvolutions indicated $\sim 21 \alpha$-helical residues for SP-Css ion-lock 1 in

646 surfactant lipids (Table 1), $\sim 11 \alpha$-helical residues are readily calculated as unexchanged for this SP-C

647 mimic at long-times (Almlen et al., 2011; Beevers \& Kukol, 2006). Importantly, similar H/D exchange

648 kinetics was earlier reported for bovine and porcine SP-C (Vandenbussche et al., 1992; Pastrana, Maulone

$649 \&$ Mendelsohn, 1991), indicating that SP-Css ion-lock 1 is accurately mimicking the ability of native SP- 
$650 \mathrm{C}$ proteins to embed into surfactant lipids as transmembrane $\alpha$-helices. In additional studies, the kinetic

651 H/D exchange profile for SP-Css ion-lock 1 was nearly identical to that of SP-C33 UCLA (Fig. 6), and

652 very similar to that reported earlier for the conventional SP-C33 (Almlen et al., 2011). The comparable

653 H/D exchange and maximum tilt angles observed for SP-Css ion-lock 1, SP-C33 UCLA and SP-C33

654 (Figs. 5 and 6; Table 1) (Almlen et al., 2011) indicate that these three SP-C mimics similarly incorporate

655 into surfactant lipids as transmembrane $\alpha$-helices. Interestingly, the H/D curve for SP-Cff ion-lock 1 in

656 Fig. 6 demonstrates both similarities and differences with those of SP-Css ion-lock 1 and SP-C33 UCLA.

657 Despite SP-Cff ion-lock 1 showing fast-, intermediate- and very slow-exchange compartments analogous

658 to those of the other two SP-C mimics, there was overall a more rapid H/D exchange for SP-Cff ion-lock 1

659 in Fig. 6. These discrepancies were particularly noticeable at $6 \mathrm{~h}$, when the unexchanged hydrogen

660 component for SP-Cff ion-lock 1 was $\sim 23.2 \%$, while those for SP-Css ion-lock 1 and SP-C33 UCLA were

661 each $\sim 51 \%$ (Fig. 6). Consequently, only $\sim 3.5$ helical residues of SP-Cff ion-lock 1 showed unexchanged

662 hydrogen at long-times in Fig. 6, compared to the $\sim 11$ helical residues that remain unexchanged in either

663 SP-Css ion-lock 1 or SP-C33 UCLA. The sharply lower unexchanged helical protons for SP-Cff ion-lock

6641 in surfactant lipids (Fig. 6) may be due to a more flexible membrane $\alpha$-helical component or increased

665 accessibility of helical amide hydrogens to the aqueous medium.

666

667

In Vitro Surface Activity Determined with Adsorption Surface Pressure Measurements

668

Synthetic surfactant preparations were formulated by mixing synthetic lipids (Lipids) consisting

669

of 5:3:2 (weight ratio) DPPC:POPC:POPG, with 3.0\% by weight SP-Css ion-lock 1, SP-Cff ion-lock 1,

670 SP-Cff, SP-C33 ion-lock 1, SP-C33 UCLA (positive control) and Lipids (negative control). Here,

671 adsorption was determined as the Adsorption Surface Pressure $(\mathrm{mN} / \mathrm{m})$ as a function of time $(0-15 \mathrm{~min})$,

672 with higher surface pressures reflecting lower surface tensions. The time-course plots in Fig. 7 indicate

673 that each of these SP-C mimics attained maximal adsorption surface pressure within $\sim 5-10 \mathrm{~min}$. The

674 relative order for the surfactant activities of the various preparations was as follows: SP-C33 ion-lock $2 \geq$

675 SP-Css ion-lock 1, SP-C33 UCLA $>>$ SP-Cff ion-lock $1 \sim$ SP-Cff $>$ Lipids. Previous in vitro studies on 
SP-C(Leu), a homologous precursor to our SP-C33 UCLA, with a lipid mixture of DPPC/POPG (7:3 ratio by mass) showed similarly enhanced adsorption surface pressure curves (Nilsson et al., 1998).

\section{In Vitro Dynamic Surface Activity Determined with Captive Bubble Surfactometry} SP-C33 ion-lock 1 surfactant exerted similar low surface tensions as SP-C33 UCLA. Contrarily, SP-Cff tension values of 19, 16, 11 and $14 \mathrm{mN} / \mathrm{m}(\mathrm{p}<0.001)$ versus SP-Css ion-lock 1 and SP-C33 UCLA after

692 ten cycles on the captive bubble surfactometer (Fig. 8). In summary, the relative order for the surfactant

693 activities determined with captive bubble surfactometry for the various preparations was as follows: SP-

694 Css ion-lock $1 \sim$ SP-C33 UCLA $\sim$ SP-C33 ion-lock $1>>$ SP-Cff $\sim$ SP-Css $>>$ Lipids $\sim$ SP-Cff ion-lock 1.

\section{In Vivo Activity of Synthetic Surfactants in Ventilated, Lung-Lavaged, Surfactant-Deficient Rabbits}


700

701

702

703

704

705

706

707

708

709

710

711

712

713

714

715

716

717

718

719

720

721

722

723

724

725

ventilated rabbits with ARDS induced by in vivo lavage (Fig. 9). Oxygenation and lung compliance increased quickly to a plateau phase (within $\sim 30-60 \mathrm{~min}$ ) after instillation of SP-Css ion-lock 1 and SPC33 UCLA, with SP-C33 ion-lock 1 reaching similarly high functional plateaus after somewhat longer times (within $\sim 60 \mathrm{~min}$ ) (Fig. 9). On the other hand, the SP-C mimics SP-Cff and SP-Cff ion-lock 1 restored more slowly (within $\sim 90 \mathrm{~min}$ ) and only partially the oxygenation and dynamic compliance observed with SP-Css ion-lock 1 and SP-C33 UCLA. The corresponding oxygenation and dynamic compliance curves obtained for SP-Css (not shown for clarity) overlapped those of SP-Cff in Fig. 9. Last, instillation of the negative control of Lipids alone had minimal effects on arterial oxygenation or compliance. The relative order of pulmonary activity in terms of both oxygenation and compliance $(\geq 90$ min) was given as: SP-Css ion-lock $1 \sim$ SP-C33 UCLA (positive control) $\sim$ SP-C33 ion-lock 1 > SP-Cff $\sim$ SP-Css $>$ SP-Cff ion-lock $1>>$ Lipids (negative control). Although the differences in oxygenation and compliance between SP-Css ion-lock 1, SP-C33 UCLA and SP-C33 ion-lock 1 were not statistically significant starting at 90 min after surfactant instillation, each of these SP-C mimics consistently exceeded the corresponding performance of SP-Cff, SP-Css, SP-Cff ion-lock 1 and lipids only surfactants $(\mathrm{p}<0.01)$ (Fig. 9).

\section{DISCUSSION}

Structural and functional studies investigated first generation SP-C peptides based on native SP-C sequences (e.g., synthetic SP-Cff and SP-Css lacking palmitoyl groups; Fig. 1), and assessed their utility as scaffolds for new SP-C ion-lock peptides with enhanced helicity and surfactant activities. Unlike the high $\alpha$-helix observed for native dipalmitoylated SP-C in surfactant lipids (Vandenbussche et al., 1992; Pastrana, Maulone \& Mendelsohn, 1991), FTIR spectroscopy for either SP-Css or SP-Cff in lipids with aqueous buffer showed primarily $\beta$-sheet (Fig. 2A; Table 1). Comparable $\beta$-structures also predominated for SP-Css and S-Cff in either 100\% aqueous buffer or 65\% HFIP/35\% aqueous buffer (Table 2), indicating that these SP-C mimic conformations are insensitive to the polarity of their environment. These FTIR spectral results are probably due to the strong parallel $\beta$-sheet propensities predicted by the PASTA 
726

727

728

729

730

731

732

733

734

735

736

737

738

739

740

741

742

743

744

745

746

747

748

749

750

751

analysis for SP-Css or SP-Cff in Fig. 3. The above findings also suggest that palmitoylcysteines in native SP-C at least partially act as helical 'adjuvants' to override the $\beta$-sheet propensities of its primary sequence (Walther et al., 2010; Conchillo-Sole et al., 2007). With this background, it was surprising that aqueous HFIP did not substantially raise the $\alpha$-helicity of SP-Css or SP-Cff over that in aqueous buffer (Table 2), especially because HFIP is a strong helix-inducing and stabilizing cosolvent (Hirota, Mizuno \& Goto, 1997) and provides an apolar environment that simulates lipid membranes (Crescenzi et al., 2002). HFIP protects against $\beta$-sheet formation by “Teflon ${ }^{\circledR}$ Coating” exposed $\alpha$-helices (Rajan et al., 1997; Crescenzi et al., 2002; Roccatano et al., 2005), thereby preventing the water attack on the helical H-bonds that are required for conversion to $\beta$-sheet. Consequently, the elevated $\beta$-sheet observed for SP-Css and SP-Cff in aqueous HFIP (Table 2) may be due to the extraordinarily high $\beta$-sheet propensities of these SPC mimics (Fig. 3) outweighing the helix-promoting actions of HFIP.

\section{Comparisons with previous FTIR results (Zandomeneghi et al., 2004; Cerf et al., 2009)}

additionally suggest that SP-Css and SP-Cff form 'amyloid-like,' $\beta$-sheet fibrils in surfactant lipids, and that these fibrils may be responsible for the low surfactant activities observed with SP-C mimics (Figs. 79). An earlier literature review indicated that native $\beta$-sheet proteins typically produce FTIR spectra with maxima at frequencies above $\sim 1630 \mathrm{~cm}^{-1}$, while the corresponding spectra of amyloid fibrils demonstrate maxima at $\sim 1630 \mathrm{~cm}^{-1}$ and lower frequencies (Zandomeneghi et al., 2004). Following this scheme, the FTIR spectra in Fig. 2A suggest that SP-Css and SP-Cff with lipids may each be forming amyloidogenic fibrils. Further comparisons with FTIR analyses of the amyloid A $\beta(1-42)$ suggested that SP-Css or SP-Cff in lipids are producing fibrils (Cerf et al., 2009). Under conditions that promote fibrils, FTIR spectra of $\mathrm{A} \beta(1-42)$ exhibited a single peak at $\sim 1630 \mathrm{~cm}^{-1}$ and negligible absorption at $\sim 1695 \mathrm{~cm}^{-1}$, and fibrils were assigned to $A \beta(1-42)$ forming parallel $\beta$-sheet. Contrarily, under conditions favoring smaller $A \beta(1-42)$ oligomers, FTIR spectra showed a primary peak at $\sim 1630 \mathrm{~cm}^{-1}$ and a significant minor peak (i.e., $\sim 1 / 5^{\text {th }}$ the intensity of the major component) that were attributed to $A \beta(1-42)$ oligomers folding like antiparallel $\beta$-sheet (Cerf et al., 2009). Because the FTIR spectra of SP-Css and SP-Cff in surfactant lipid (Fig. 2A) are most comparable to those of fibrillar $\mathrm{A} \beta(1-42)$ (Cerf et al., 2009)[68], our SP-C mimics in surfactant 
752 lipids may similarly adopt parallel $\beta$-sheet, fibril structures. This assignment agrees with PASTA

753 predictions that the C-terminal regions of SP-Css and SP-Cff have high parallel $\beta$-sheet propensities (Figs.

7543 and 4). Moreover, prior experiments indicate that the homologous rSP-C (Fig. 1) readily transforms

755 from $\alpha$-helical conformers to $\beta$-sheet fibrils in lipid-mimics (Luy et al., 2004). Any $\beta$-sheet fibrils

756 occurring in lipid mixtures of SP-Css or SP-Cff would not necessarily be toxic but instead may represent

757 an inert peptide reservoir. Recent evidence with the amyloid A $\beta$ protein demonstrates that it is only small

758 molecular weight oligomers, but not monomers or high molecular weight fibrils, that are cytotoxic (Haas

759 \& Selkoe, 2007; Nerelius, Fitzen \& Johansson, 2010). In this context, SP-Cff and/or SP-Css in lipids

760 showed surfactant activities that were between those of lipids alone (negative control) and those with the

761 more active SP-C33 UCLA (positive control) (see below and Figs. 7-9). The relatively low surfactant

762 activities for SP-Cff and SP-Css with in vitro (Figs. 7 and 8) and in vivo (Fig. 9) assays may be due to

763 these SP-C mimics forming inactive fibrils consisting of parallel $\beta$-sheet, thus effectively lowering the

764 concentrations of surface-active SP-C peptides in helical conformations.

765

Because the above first generation SP-C peptides were unsuccessful in reproducing key structural

766 and functional properties of the native protein in surfactant lipids, a second generation of SP-C mimics without palmitoyls is now being developed that seeks to increase $\alpha$-helicity and surfactant activities through amino acid replacements. Previous experimental and theoretical studies indicated that SP-C embeds deeply into lipid bilayers, in which its helical axis and covalently attached palmitoyls are nearly parallel to the fatty acyl chains, while its cationic $\mathrm{N}$-terminal region is restricted to the polar headgroup region (see Introduction). As enhanced in vitro and in vivo surfactant activities are highly correlated with

772 elevated $\alpha$-helix content, a biomimetic approach was used to design $\alpha$-helicity and other essential

773 molecular features of native SP-C into new SP-C analogs. One such class of SP-C mimics is the SP-C33

774 peptide, produced with the porcine SP-C sequence as a template, in which ten valines were replaced by

775 leucines, the two palmitoylcysteines substituted with serines and the $\mathrm{N}$-terminal arginine was moved

776 closer to the poly-Leu sequence (Fig. 1) (Johansson et al., 2003). This synthetic SP-C mimic showed in 777 vitro and in vivo surfactant activities equivalent to those of native SP-C (Johansson et al., 2003; Almlen et 
778

779

780

781

782

783

784

785

786

787

788

789

790

791

792

793

794

795

796

797

798

799

800

801

802

803

al, 2011), which may be due to both SP-C33 and native SP-C similarly inserting into surfactant lipids with slightly tilted transmembrane $\alpha$-helices (Pastrana, Maulone \& Mendelsohn, 1991;Vandenbussche et al., 1992; Almlen et al., 2011).

We performed experiments here to determine whether an SP-C33 peptide with high surfactant activity was appropriate as a positive control to benchmark the structural and surfactant properties of other second generation SP-C peptides. With respect to the SP-C33 class, studies were conducted with 'SP-C33 UCLA,' an SP-C mimic with the same sequence as SP-C33 (Fig. 1) but synthesized using a separate protocol. For example, FTIR spectra of SP-C33 UCLA in aqueous buffer, HFIP-aqueous buffer and surfactant lipids in aqueous buffer indicated that the predominant conformation was $\alpha$-helix (Fig. 2B; Tables 1 and 2). These spectral results agree with prior findings showing high $\alpha$-helix for SP-C33 in surfactant lipids and lipid-mimics (i.e., methanol or detergent micelles) using CD or FTIR spectroscopy (Johansson et al., 2003; Almlen et al., 2011). The enhanced $\alpha$-helix levels for SP-C33 UCLA and SP-C33 are consistent with PASTA predictions that SP-C33 sequences (Fig. 1) have a lower propensity to form $\beta$ sheet than native SP-C peptides (Figs. 3 and 4). The total replacement of the poly-valine sequence with poly-leucine in SP-C33 UCLA suppresses 'amyloid-like' $\beta$-sheet structures by favoring $\alpha$-helix and eliminates the unstable conformations associated with SP-C lacking palmitoyl groups. Orientation FTIR and H/D exchange experiments indicated that SP-C33 UCLA incorporates into lipid bilayers as a slightly tilted transmembrane $\alpha$-helix (Figs. 5 and 6; Table 1), demonstrating that SP-C33 UCLA accurately mimics the membrane topography of both SP-C33 (Almlen et al., 2011) and native dipalmitoylated SP-C (Pastrana, Maulone \& Mendelsohn, 1991; Vandenbussche et al., 1992). Also supporting the above experimental results are MPEx hydropathy predictions that SP-C33 sequences insert into membrane bilayers when folded as $\alpha$-helices (Fig. 4). In this context, note that SP-C33 UCLA demonstrated high in vitro (Figs. 7 and 8) and in vivo (Fig. 9) surfactant activities, consistent with the elevated surfactant activities reported earlier for SP-C33 in comparisons with commercial preparations containing native SPC (Johansson et al., 2003; Almlen et al., 2011). The tilted transmembrane $\alpha$-helix, identified here for SPC33 UCLA in lipid bilayers (Figs. 5 and 6; Table 1) and earlier for SP-C33 (Almlen et al., 2011), likely 
804

805

806

807

808

809

810

811

812

813

814

815

816

817

818

819

820

821

822

823

824

825

826

827

828

829

represents the surface-active arrangement shared by native SP-C, and departures from this membrane topography may reduce surfactant properties.

The principal hypothesis to be tested in this paper was that the selective insertion of salt-bridges may produce SP-C mimics that insert into lipid bilayers as slightly tilted, transmembrane $\alpha$-helices with high surfactant activity. In partial support of this hypothesis, prior reports indicate that engaged saltbridges (or ion-locks) in either designer peptides or native membrane proteins promote $\alpha$-helix. With CD studies of aqueous synthetic peptides, for example, incorporation of $\mathrm{Glu}^{-}$and $\mathrm{Lys}^{+}, \mathrm{Asp}^{-}$and $\mathrm{Lys}^{+}, \mathrm{Asp}^{-}$and $\mathrm{Arg}^{+}$or $\mathrm{Glu}^{-}$and $\mathrm{Arg}^{+}$spaced at $(i+4)$ intervals in the sequence was helix stabilizing (Marqusee \& Baldwin, 1987). This enhanced $\alpha$-helicity for aqueous peptides is at least partly due to the formation of an electrostatically neutral ion-pair via the positive- and negative-side groups for the various amino-acid pairings (Kumar \& Nussinov, 1999 \& 2002). Employing an augmented Wimley-White (WW) hydrophobicity scale, MPEx analyses predicted that 'ion-locks' such as Glu'- Lys $^{+}$increase the helicity and lipid partitioning of peptides into membranes (Snider et al., 2009). Additionally, continuum electrostatic calculations indicated that salt-bridges with close distances $(\leq 4.0 \AA)$ between the cationic residue "N" and the nearest anionic residue "O" are mostly $\alpha$-helix stabilizing, while those with correspondingly further distances (> 5.0 §) may be helix destabilizing (Kumar \& Nussinov, 1999 \& 2002). Earlier surveys of PDB-deposited structures have reported salt-bridges with a close-range cutoff $(\leq$ $4.0 \AA \AA$ ) in numerous proteins (Kumar \& Nussinov, 1999 \& 2002) including $\mathrm{Ca}^{2+}$-ATPase (Toyoshima et al., 2000) (PDB: 1SU4) with a membrane helix stabilized by an intrahelical charge-pair (e.g., Asp ${ }^{-}-59-\mathrm{Arg}^{+}-$ 63) when facing lipids (Bano-Polo et al., 2012). Importantly, ion-locks have been used in several designer peptides as a 'Molecular Velcro®' for specific purposes, such as enhancement of $\alpha$-helix and anti-HIV activity in sifuvirtide (He et al., 2008), and the trapping of otherwise unstable structural features such as $\pi$ helix (Chapman et al., 2008) and the second $\beta$-hairpin of the B1 domain of protein G (HuyghuesDespointes et al., 2006). 
locks) into SP-C sequences lacking palmitoyl groups. With a charged ion-pair in the midsection of the

831 parent SP-Css (Fig. 1), SP-Css ion-lock 1 in either lipids, aqueous HFIP or aqueous buffer remarkably

832 shifted its principal conformation to $\alpha$-helix from amyloid-like $\beta$-sheet (Fig. 2; Tables 1 and 2). In

833 particular, FTIR spectral deconvolutions for SP-Css ion-lock 1 in surfactant lipids showed enhanced $\alpha$ -

834 helix similar to that determined for SP-C33 UCLA, our positive control SP-C peptide (i.e., 63.1 vs. $66.9 \%$,

835 respectively; Table 1). Oriented FTIR and H/D exchange experiments for SP-Css ion-lock 1 indicated a

836 slightly tilted transmembrane $\alpha$-helix in surfactant lipid bilayers (Figs. 5 and 6; Table 1), consistent with

837 MPEx hydropathy predictions for this SP-C mimic with a neutralized charged ion-pair (Fig. 4). A slightly

838 tilted, transmembrane $\alpha$-helix was also identified for SP-C33 UCLA comparable to that of SP-Css ion-

839 lock 1 (Figs. 5 and 6; Table 1). In this context, note that SP-Css ion-lock 1 showed high surfactant

840 activities equal to SP-C33 UCLA, as measured with in vitro adsorption surface pressure (Fig. 7) and

841 minimum surface tension (Fig. 8), and also with in vivo oxygenation and dynamic compliance

842 experiments of surfactant-treated, ventilated rabbits with ARDS (Fig. 9). One explanation for the

843 enhanced activities of SP-Css ion-lock 1 is that a close-range salt-bridge (Kumar \& Nussinov, 1999 \&

844 2002) forms between the Glu- -20 and $\mathrm{Lys}^{+}-24$ residues, which promotes accurate mimicking of the high $\alpha-$

845 helicity and the membrane topography of SP-C33 UCLA (Fig. 2; Tables 1 and 2), SP-C33 (Johansson et

846 al., 2003; Almlen et al., 2011) or native SP-C (Pastrana, Maulone \& Mendelsohn, 1991; Vandenbussche et

847 al., 1992). Analogous close-range salt-bridges occurring for the Glu-20 and $\mathrm{Lys}^{+}-24$ pair might also

848 account for the high $\alpha$-helix seen for SP-Css ion-lock 1 in either aqueous buffer or aqueous HFIP buffer

849 (Table 2).

850

Experiments next evaluated correlations between $\alpha$-helicity, lipid bilayer topography and

851 surfactant properties for the SP-Cff ion-lock 1 mimic. Although the insertion of the $\mathrm{Glu}^{-}-\mathrm{Lys}^{+}$pairing into

852 SP-Cff primarily converted the SP-Cff ion-lock 1 in lipids from $\beta$-sheet to $\alpha$-helix (Fig. 2A), FTIR

853 deconvolutions of SP-Cff ion-lock 1 indicated lower $\alpha$-helix (i.e., 44.6\%) than the corresponding $\alpha$-helix

854 for SP-Css ion-lock 1 (63.1\%) and SP-C33 UCLA (67.2\%) (Table 1). Oriented FTIR and H/D exchange

855 studies of SP-Cff ion-lock 1 showed that the $\alpha$-helix incorporates poorly into the lipid bilayer (Fig. 6), and 
856 also is more tilted $\left(34.2^{\circ}\right)$ to the membrane normal than those of either SP-Css ion-lock $1\left(22.6^{\circ}\right)$ or SP-

857 C33 UCLA $\left(22.4^{\circ}\right)$ (Fig. 5; Table 1). Because of its shortened C-terminal helix and much greater tilt, the $\alpha-$

858 helix of SP-Cff ion-lock 1 may thus be restricted to a single monolayer. This abnormal membrane

859 topography may be partially due to SP-Cff ion-lock 1 forming a disruptive, long-range salt-bridge (Kumar

$860 \&$ Nussinov, 2002) between the Glu- -20 and $\mathrm{Lys}^{+}-24$ residues, which would be expected to reduce both

861 helix stability (Table 1) and lipid affinity (Fig. 4). The exposure of the charged side-chain groups

862 occurring in long-range salt-bridges (Kumar \& Nussinov, 2002) may promote electrostatic interactions

863 between the Glu-20 and Lys ${ }^{+}-24$ residues of SP-Cff ion-lock 1 and other membrane components,

864 including Lys ${ }^{+}-24$ binding to anionic phosphates in the polar headgroup of POPG. Such detergent-like

865 properties may be responsible for the low in vitro and in vivo surfactant activities obtained for

866 formulations containing SP-Cff ion-lock 1, when compared with preparations containing SP-C33 UCLA,

867 SP-Css ion-lock 1 or the parent SP-Cff (Figs. 7-9). Interestingly, SP-Cff ion-lock 1 in aqueous buffer

868 additionally showed much lower $\alpha$-helix and higher loop-turns than those corresponding levels in SP-Css

869 ion-lock 1 (Table 2), suggesting that replacement of the vicinal serines with phenylalanines may have also

870 produced a long-range salt-bridge that was destabilizing in an aqueous environment.

In contrast to the surfactant properties of SP-Cff ion-lock 1, preparations containing SP-C33 ion-

872 lock 1 showed elevated surface activity using captive bubble surfactometry (Fig. 8), and also high arterial 873 oxygenation and dynamic compliance in surfactant-treated, ventilated rabbits with ARDS (Fig. 9). The 874 enhanced in vitro and in vivo surfactant activities of SP-C33 ion-lock 1 match those of SP-Css ion-lock 1 875 and SP-C33 UCLA (Figs. 8 and 9), and are likely due to all three SP-C mimics exhibiting comparable $\alpha$ 876 helicity in lipids (Fig. 2; Table 1) and incorporating as tilted, transmembrane $\alpha$-helices (Figs. 5 and 6;

877 Table 1). For deep insertion into lipid bilayers as a helical transmembrane $\alpha$-helix, MPEx calculations in 878 Fig. 4 additionally suggested that SP-C33 ion-lock 1will form a close-range salt-bridge in the membrane

879 interior. Interestingly, this electrostatically neutral ion-pair is unable to further increase the $\alpha$-helix content

880 and surfactant activity of SP-C33 ion-lock 1 over those observed with the control SP-C33 UCLA,

881 probably because these properties are already optimized in the host SP-C33 UCLA peptide. Nevertheless, 
882

883

884

885

886

887

888

889

890

891

892

893

894

895

896

897

898

899

900

901

902

903

904

905

906

907

the presence of a close-range salt-bridge in the mid-section of SP-C33 ion-lock 1 may improve both the thermostability (Kumar, Tsai \& Nussinov, 2000; Kumar \& Nussinov, 2002) and storage properties of this SP-C mimic over those of its parent SP-C33 UCLA.

Recent Molecular Dynamics (MD) simulations were also performed to assess further whether the enhanced surfactant activities for SP-Css ion-lock 1 were due to a close-range salt-bridge between Glu-20 and Lys ${ }^{+}-24$ that stabilizes the $\alpha$-helix (AJ Waring, FJ Walther, KJ Longmuir, LM Gordon, unpublished data, 2014). MD runs using GROMACS (http://www.gromacs.org) should provide valuable information on the detailed 3D-structures of SP-C mimics in the bilayer (Walther et al., 2010; Schwan et al., 2011). For simulations of SP-Css ion-lock 1 in a lipid bilayer-water box, the " 0 nsec" model was obtained by first templating its primary sequence (Fig. 1) onto the 2D-NMR structure of porcine SP-C (Johansson et al., 1994) (PDB: 1SPF). The homology modelled SP-Css ion-lock 1 was centered in a bilayer box with its helical axis normal to the membrane surface, then surrounded by 29 POPG lipids interacting with the transmembrane peptide as boundary lipid, and finally 79 DPPC lipids and 18 POPC lipids added to fill out the bilayer. In the GROMACS Version 4.5.5 environment, MD simulations (100 nsec) for the SP-Css ionlock 1-lipid ensemble in a water box using the ffG53a6 force-field rapidly reached equilibrium $(\leq \sim 60-70$ nsec). The equilibrated "100 nsec" model shows that the hydrophobic C-terminus of SP-Css ion-lock 1 inserts as a transmembrane $\alpha$-helix (residues $\sim 7-19$ and $\sim 22-32$ ) at a slight tilt (i.e., $\theta$ ) to the normal of the bilayer surface, and also the polar N-terminus (residues $~ 1-6$ ) interacts as an extended random-coil with polar lipid headgroups. In particular, the helical axis for the "100 nsec" SP-Css ion-lock 1 is tilted at an angle (i.e., $\theta \sim 25^{\circ}$ ) from the normal to the membrane surface that is in agreement with the maximum tilt angle $(\Theta)$ from oriented FTIR spectra (i.e., $\sim 23^{\circ}$ ) (see Table 1). The similarity in the membrane insertion angles for helical SP-Css ion-lock 1 obtained from MD simulation and polarized FTIR argues that the peptide-lipid bilayers are deposited as well-ordered multilayers on the germanium crystal with minimal unoriented (isotropic) peptide. Substantial amounts of isotropic peptide would be expected to reduce the dichroic ratio $\mathrm{R}$ and increase $\Theta$ over the actual tilt angle $\theta$, which is not observed in our MD simulation and polarized FTIR experiments (Fig. 5; Table 1) (Beevers \& Kukol, 1999). The MD simulations and 
908 "100 nsec" models for SP-Css ion-lock 1, SP-C33 ion-lock 1 and SP-C33 are all similar, with the three

909 SP-C mimics indicating comparable MD tilt angles $\left(\sim 20-25^{\circ}\right)$ for their long $(\sim 22$-residues $)$

910 transmembrane $\alpha$-helices that match their polarized FTIR tilt angles $\left(\Theta=\sim 23^{\circ}\right)$ (see Table 1). Importantly,

911 close-range salt-bridges were identified from the "100 nsec" models for SP-Css ion-lock 1 and SP-C33

912 ion-lock 1 in the surfactant lipid bilayer, with gaps of 3.9 and $3.3 \AA$ between the respective Lys ${ }^{+}-24$ "N"

913 and Glu'- 20 "O”" pairs (Kumar \& Nussinov, 1999; Kumar \& Nussinov, 2002) (see Introduction). The salt-

914 bridges for the SP-C mimics each lie at the center of the surfactant lipid bilayer, which is consistent with

915 MPEx calculations indicating that SP-C peptides bearing ion-locks may readily embed into the lipid

916 bilayer as $\alpha$-helices (Fig. 4). The relatively low H/D exchange for the $\alpha$-helical residues of either SP-C33

917 UCLA or SP-Css ion-lock 1 over $\sim 6 \mathrm{hr}$ (Fig. 6) is also probably due to the deep penetration of these SP-C

918 mimics into lipid bilayers as transmembrane $\alpha$-helices. Consequently, the high in vitro and in vivo

919 surfactant activities reported here for SP-Css ion-lock 1, SP-C33 UCLA and SP-C33 ion-lock 1 (Figures

920 7-9) are probably due to each of these SP-C mimics reproducing key structural features of native SP-C

921 bound to lipid bilayers (see Introduction).

922

Analogous MD simulations were also conducted to determine whether the reduced surfactant

923

activities of SP-Cff ion-lock 1 reported here were due to a long-range salt-bridge forming between Glu-20

924 and Lys ${ }^{+}-24$ that destabilizes the $\alpha$-helix (AJ Waring, FJ Walther, KJ Longmuir, LM Gordon, unpublished

925 data, 2014). Although the Glu- - Lys $^{+}$pair converts SP-Cff to principally $\alpha$-helical SP-Cff ion-lock 1 (Fig.

926 2A; Tables 1 and 2), FTIR deconvolutions of SP-Cff ion-lock 1 in lipids indicated lower $\alpha$-helix (i.e.,

$92744.6 \%)$ than the $\alpha$-helix for SP-Css ion-lock 1 (63.1\%) or SP-C33 UCLA (67.2\%). Oriented FTIR and

$928 \mathrm{H} / \mathrm{D}$ exchange studies on SP-Cff ion-lock 1 further showed that its short $\alpha$-helix ( $\sim 15$ residues) poorly

929 incorporated in the bilayer (Fig. 6), and also had a maximum tilt angle $\left(\Theta=34.2^{\circ}\right)$ greater than those of

930 SP-Css ion-lock 1 or SP-C33 UCLA (Fig. 5; Table 1). MD simulations confirmed that the "100 nsec" SP-

931 Cff ion-lock 1 restricted its shortened C-terminal $\alpha$-helix to one leaflet with a tilt $\left(\theta \sim 36^{\circ}\right)$ that matched the

932 maximum tilt angle from polarized FTIR experiments. This abnormal membrane topography is partially

933 due to SP-Cff ion-lock 1 forming a disruptive, long-range salt-bridge ( $8.9 \AA$ ) between the Glu-20 and 
934 Lys $^{+}-24$ residues (Kumar, Nussinov, 2002), which may reduce helix stability (Fig. 6) and lower lipid

935 affinity (Fig. 4). The exposure of the charged side-chain groups occurring in long-range salt-bridges may

936 promote electrostatic interactions between either $\mathrm{Glu}^{-}-20$ or $\mathrm{Lys}^{+}-24$ with other membrane components

937 (Kumar \& Nussinov, 2002), including Lys ${ }^{+}-24$ binding to anionic phosphates in the polar headgroup of

938 POPG. The trigger for the above "detergent-like" actions with SP-Cff ion-lock 1 is the replacement of the

939 vicinal serines with phenylalanines. These substitutions significantly increase the hydrophobicity of the N-

940 terminal domain of SP-Cff ion-lock 1 over that of SP-Css ion-lock 1, thereby permitting deeper insertion

941 of the N-terminus into the fatty-acyl regions of the bilayer and 'snorkeling' of the C-terminal helix into the

942 opposing monolayer. Such detergent-like properties may be responsible for the low in vitro and in vivo

943 surfactant activities obtained for formulations containing SP-Cff ion-lock 1, when compared with

944 preparations containing SP-C33 UCLA, SP-Css ion-lock 1 or SP-Cff (Figs. 7-9).

945

946

CONCLUSIONS

Results from experiments on our suite of SP-C peptides confirm that a selective incorporation of

948 salt-bridges produces SP-C mimics that insert into lipid bilayers as slightly tilted, transmembrane $\alpha$ -

949 helices with high surfactant activity. Our lead peptide is SP-Css ion-lock 1, an SP-C mimic that shows

950 enhanced in vitro and in vivo surfactant activities over its parent SP-Css by readily inserting into lipid

951 bilayers as an alpha-helix that is slightly tilted and spans both monolayers. Importantly, SP-Css ion-lock 1

952 simulates well both the structural and functional properties of SP-C33 UCLA, a member of the SP-C33

953 class of SP-C mimics. Our working hypothesis is that the SP-Css ion-lock 1 in lipids improves its

954 surfactant functions by stabilizing $\alpha$-helix through a close-range salt-bridge between the $\mathrm{Glu}^{-}-20$ and Lys ${ }^{+}-$

95524 residues. As a further test of this proposition, more detailed 3D information on the structure and

956 membrane topography of SP-C ion-locks will be obtained using ${ }^{13}$ C-FTIR spectroscopy (Gordon et al.,

957 2000; Waring et al., 2005), 2D-NMR spectrometry (Sarker et al., 2007) and/or all-atom MD simulation

958 techniques (Walther et al., 2010; Almlen et al., 2010). Additionally, polarized FTIR spectra of SP-C

959 mimics in surfactant lipids should indicate the fatty-acyl chain orientation with respect to the peptide 
960 helical axis, and likewise show if there are any protein-induced perturbations in lipid conformations

961 (Clercx et al., 1995). SP-C ion-lock peptides will also be modified to determine whether their surfactant

962 properties may be further optimized. Because the palmitoyls of native SP-C have been proposed to

963 increase surfactant activity by physically coupling stacked lipids to the monolayer (Leonenko et al., 2007),

964 future atomic force microscopy (AFM) (Ding et al., 2001; Frey et al., 2010) and functional studies may

965 assess if dipalmitoylated SP-Css ion-lock 1 similarly shows enhanced properties through an analogous

966 mechanism. Surface plasmon resonance (SPR) studies may also indicate if salt-bridges affect either the

967 self-association of SP-C ion-locks or the binding of SP-C ion-locks to SP-B mimics or lipid ensembles

968 (Walther et al., 2010).

969 Lastly, in vivo experiments will determine whether synthetic preparations containing SP-B

970 analogs and SP-C ion-locks may be superior to single-peptide surfactants in the treatment of NRDS and

971 ALI/ARDS. In earlier collaborative studies (Almlen et al., 2010), we investigated the in vivo activities of

972 Mini-B (i.e., MB, a 34-residue 'short-cut' version of SP-B with an engineered turn linking the N- and C-

973 terminal helical domains) and/or SP-C33 with synthetic lipids in preterm newborn rabbits. Treatment with

974 either Mini-B or SP-C33 increased tidal and lung gas volumes, while combination therapy demonstrated

975 an additive effect in this validated animal model for NRDS (Almlen et al., 2010). More recently, animal

976 models of lung-lavaged ARDS and chemically induced ALI were treated with Super Mini-B (i.e., SMB, a

977 41-residue truncated version of SP-B, with the seven-residue N-terminus of SP-B added to the N-terminus

978 of Mini-B) and SP-C33 UCLA. Synthetic formulations using either SMB or SP-C33 UCLA improved

979 blood oxygenation and dynamic compliance in these ARDS/ALI models, while combination therapy

980 indicated an additive effect (Walther et al., 2014). Similar in vivo experiments in which the SP-C33 class

981 is replaced with SP-Css ion-lock 1 may show if SP-C mimics with salt-bridges may also be therapeutically

982 beneficial.

983

984 LIST OF ABBREVIATIONS 


\begin{tabular}{|c|c|c|}
\hline 985 & ALI & acute lung injury \\
\hline 986 & ARDS & acute respiratory distress syndrome \\
\hline 987 & Asp & aspartic acid \\
\hline 988 & Arg & arginine \\
\hline 989 & ATR & attenuated total reflectance \\
\hline 990 & $\mathrm{CD}$ & circular dichroism \\
\hline 991 & DPPC & dipalmitoyl phosphatidylcholine \\
\hline 992 & $\mathrm{H} / \mathrm{D}$ & hydrogen/deuterium \\
\hline 993 & HFIP & hexafluoroisopropanol \\
\hline 994 & HPLC & high performance liquid chromatography \\
\hline 995 & FTIR & Fourier transform infrared spectroscopy \\
\hline 996 & Glu & glutamate \\
\hline 997 & Lys & lysine \\
\hline 998 & MPEx & membrane protein explorer \\
\hline 999 & NRDS & neonatal respiratory distress syndrome \\
\hline 1000 & 2D-NMR & two-dimensional nuclear magnetic resonance spectroscopy \\
\hline 1001 & $\mathrm{PaO} 2$ & partial pressure of $\mathrm{O} 2$ in arterial blood \\
\hline 1002 & $\mathrm{PaCO} 2$ & partial pressure of $\mathrm{CO} 2$ in arterial blood \\
\hline 100 & PASTA & prediction of amyloid structure aggregation \\
\hline
\end{tabular}


1004 POPC palmitoyl-oleoyl phosphatidylcholine

1005 POPG palmitoyl-oleoyl phosphatidylglycerol

1006 SP-B surfactant protein B

1007 SP-C surfactant protein C

1008 WW Wimley-White

1009

1010 REFERENCES

1011 Almlen A, Walther FJ, Waring AJ, Robertson B, Johansson J, Curstedt T. 2010. Synthetic surfactant based

1012 on analogues of SP-B and SP-C is superior to single-peptide surfactants in ventilated premature

1013 rabbits. Neonatology 98: 91-99.

1014 Almlen A, Vandenbussche G, Linderholm B, Haegerstrand-Bjorkman M, Johansson J, Curstedt T. 2011.

1015 Alterations of the C-terminal end do not affect in vitro or in vivo activity of surfactant protein C

1016 analogs. Biochim Biophys Acta 1818: 27-32.

1017 Alonso C, Zasadzinski JA. 2004. Linear dependence of surface drag on surface viscosity. Phys Rev E Stat

$1018 \quad$ Nonlin Soft Matter Phys 69: 021602.

1019 Arkin IT, MacKenzie KR, Brunger AT. 1997. Site-directed dichroism as a method for obtaining rotational

1020 and orientational constraints for oriented polymers. J Am Chem Soc 119: 8973-8980.

1021 Baatz JE, Smyth KL, Whitsett JA, Baxter C, Absolom DR. 1992. Structure and functions of a dimeric

1022 form of surfactant protein SP-C: a Fourier transform infrared and surfactometry study. Chem Phys

1023 Lipids 63: 91-104.

1024 Bano-Polo M, Baeza-Delgado C, Orzaez M, Marti-Renom MA, Abad C, Mingarro I. 2012.

1025 Polar/Ionizable residues in transmembrane segments: effects on helix-helix packing. PLoS One 7:

1026 e44263. 
1027 Baoukina S, Tieleman DP. 2010. Direct simulation of protein-mediated vesicle fusion: lung surfactant 1028 protein B. Biophys J 99: 2134-2142.

1029 Baumgart F, Ospina OL, Mingarro I, Rodriguez-Crespo I, Perez-Gil J. 2010. Palmitoylation of pulmonary

1030 surfactant protein SP-C is critical for its functional cooperation with SP-B to sustain compression/ 1031 expansion dynamics in cholesterol-containing surfactant films. Biophys $J$ 99: 3234-3243.

1032 Beevers AJ, Kukol A. 2006. Secondary structure, orientation, and oligomerization of phospholemman, a 1033 cardiac transmembrane protein. Protein Sci 15: 1127-1132.

1034 Bridges JP, Wert SE, Nogee LM, Weaver TE. 2003. Expression of a human surfactant protein C mutation 1035 associated with interstitial lung disease disrupts lung development in transgenic mice. J Biol Chem $1036 \quad 278: 52739-52746$

1037 Bridges JP, Xu Y, Na CL, Wong HR, Weaver TE. 2006. Adaptation and increased susceptibility to 1038 infection associated with constitutive expression of misfolded SP-C. J Cell Biol 172: 395-407.

1039 Brower RG, Fessler HE. 2011. Another "negative" trial of surfactant. Time to bury this idea? Am J Respir $1040 \quad$ Crit Care Med 183: 966-968.

1041 Byler DM, Susi H. 1986. Examination of the secondary structure of protein by deconvolved FTIR spectra. $1042 \quad$ Biopolymers 25: 469-487.

1043 Cerf E, Sarroukh R, Tamamizu-Kato S, Breydo L, Derclaye S, Dufrene YF, Narayanaswami V, 1044 Goormaghtigh E, Ruysschaert J-M, Raussens V. 2009. Antiparallel beta-sheet: a signature structure of 1045 the oligomeric amyloid beta-peptide. Biochem J 421: 415-423.

1046 Chapman R, Kulp JL, III, Patgiri A, Kallenbach NR, Bracken C, Arora PS. 2008. Trapping a folding 1047 intermediate of the alpha-helix: stabilization of the pi-helix. Biochemistry 47: 4189-4195.

1048 Chia CS, Torres J, Cooper MA, Arkin IT, Bowie JH. 2002. The orientation of the antibiotic peptide 1049 maculatin 1.1 in DMPG and DMPC lipid bilayers. Support for a pore-forming mechanism. FEBS Lett $1050 \quad 512: 47-51$.

1051 Clark JC, Wert SE, Bachurski CJ, Stahlman MT, Stripp BR, Weaver TE, Whitsett JA. 1995. Targeted 1052 disruption of the surfactant protein B gene disrupts surfactant homeostasis, causing respiratory failure 1053 in newborn mice. Proc Natl Acad Sci USA 92: 7794-7798. 
1054

1055

1056

1057

1058

1059

1060

1061

1062

1063

1064

1065

1066

1067

1068

1069

1070

1071

1072

1073

1074

1075

1076

1077

1078

Clercx A, Vandenbussche G, Curstedt T, Johansson J, Jornvall H, Ruysschaert JM. 1995. Structural and functional importance of the C-terminal part of the pulmonary surfactant polypeptide SP-C. Eur J Biochem 229: 465-472.

Conchillo-Sole O, de Groot NS, Aviles FX, Vendrell J, Daura X, Ventura S. 2007. AGGRESCAN: a server for the prediction and evaluation of "hot spots" of aggregation in polypeptides. BMC Bioinformatics 8: 65.

Crescenzi O, Tomaselli S, Guerrini R, Salvadori S, D'Ursi AM, Temussi PA, Picone D. 2002. Solution structure of the Alzheimer amyloid b-peptide (1-42) in an apolar microenvironment. Similiarity with a viral fusion domain. Eur J Biochem 269: 5642-5648.

Curstedt T, Johansson J, Persson P, Eklund A, Robertson B, Lowenadler B, Jornvall H. 1990. Hydrophobic surfactant-associated polypeptides: SP-C is a lipopeptide with two palmitoylated cysteine residues, whereas SP-B lacks covalently linked fatty acyl groups. Proc Natl Acad Sci U S A 87: 2985-2989.

Curstedt T, Calkovska A, Johansson J. 2013. New generation synthetic surfactants. Neonatology 103: 327330.

Ding J, Takamoto DY, von NA, Lipp MM, Lee KY, Waring AJ, Zasadzinski JA. 2001. Effects of lung surfactant proteins, SP-B and SP-C, and palmitic acid on monolayer stability. Biophys J 80: 22622272.

Dluhy RA, Shanmukh S, Leapard JB, Kruger P, Baatz JE. 2003. Deacylated pulmonary surfactant protein SP-C transforms from alpha-helical to amyloid fibril structure via a $\mathrm{pH}$-dependent mechanism: an infrared structural investigation. Biophys $J$ 85: 2417-2429.

Duncan SL, Larson RG. 2010. Folding of lipid monolayers containing lung surfactant proteins SP-B(1-25) and SP-C studied via coarse-grained molecular dynamics simulations. Biochim Biophys Acta 1798: $1632-1650$.

Fields CG, Lloyd DH, Macdonald RL, Ottenson KM, Noble RL. 1991. HBTU activation for automated Fmoc solid-phase peptide synthesis. Pept Res 4: 95-101. 
1079 Frey SL, Pocivavsek L, Waring AJ, Walther FJ, Hernandez-Juviel JM, Ruchala P, Lee KY. 2010.

1080 Functional importance of the NH2-terminal insertion sequence of lung surfactant protein B. Am J

$1081 \quad$ Physiol Lung Cell Mol Physiol 298: L335-L347.

1082 Glasoe PK, Long FA. 1960. Use of glass electrodes to measure acidities in deuteriium oxide. $J$ Phys Chem $1083 \quad 64: 188-190$.

1084 Goerke J. 1998. Pulmonary surfactant: functions and molecular composition. Biochim Biophys Acta 1408: $1085 \quad 79-89$.

1086 Gordon LM, Horvath S, Longo ML, Zasadzinski JA, Taeusch W, Faull K, Leung C, Waring AJ. 1996.

1087 Conformation and molecular topography of the N-terminal segment of surfactant protein B in $1088 \quad$ structure-promoting environments. Protein Sci 5: 1662-1675.

1089 Gordon LM, Lee KYC, Lipp MM, Zasadzinski JA, Walther FJ, Sherman MA, Waring AJ. 2000.

1090 Conformational mapping of the N-terminal segment of surfactant protein B in lipid using 13C-

1091 enhanced Fourier transform infrared spectroscopy. J Peptide Res 55: 330-347.

1092 Gordon LM, Nisthal A, Lee AB, Eskandari S, Ruchala P, Jung CL, Waring AJ, Mobley PW. 2008.

1093 Structural and functional properties of peptides based on the N-terminus of HIV-1 gp41 and the C-

1094 terminus of the amyloid-beta protein. Biochim Biophys Acta 1778: 2127-2137.

1095 Grimard V, Vigano C, Margolles A, Wattiez R, van Veen HW, Konings WN, Ruysschaert JM,

1096 Goormaghtigh E. 2001. Structure and dynamics of the membrane-embedded domain of LmrA

1097 investigated by coupling polarized ATR-FTIR spectroscopy and (1)H/(2)H exchange. Biochemistry

$1098 \quad 40: 11876-11886$.

1099 Gustafsson M, Thyberg J, Naslund J, Eliasson E, Johansson J. 1999. Amyloid fibril formation by

1100 pulmonary surfactant protein C. FEBS Lett 464: 138-142.

1101 Haass C, Selkoe DJ. 2007. Soluble protein oligomers in neurodegeneration: lessons from the Alzheimer's

1102 amyloid beta-peptide. Nat Rev Mol Cell Biol 8: 101-112.

1103 He Y, Xiao Y, Song H, Liang Q, Ju D, Chen X, Lu H, Jing W, Jiang S, Zhang L. 2008. Design and

1104 evaluation of sifuvirtide, a novel HIV-1 fusion inhibitor. J Biol Chem 283: 11126-11134. 
1105 Hirota N, Mizuno K, Goto Y. 1997. Cooperative alpha-helix formation of beta-lactoglobulin and melittin

1106 induced by hexafluoroisopropanol. Protein Sci 6: 416-421.

1107 Huyghues-Despointes BMP, Qu X, Tsai J, Scholtz JM. 2006. Terminal ion pairs stabilize the second beta-

1108 hairpin of the B1 domain of protein G. Proteins 63: 1005-1017.

1109 Ikegami M, Jobe AH. 1998. Surfactant protein-C in ventilated premature lamb lung. Pediatr Res 44: 860$1110 \quad 864$.

1111 Jackson M, Mantsch HH. 1995. The use and misuse of FTIR spectroscopy in the determination of protein 1112 structure. Crit Rev Biochem Mol Biol 30: 95-120.

1113 Jayasinghe S, Hristova K, White SH. 2001. Energetics, stability, and prediction of transmembrane helices. $1114 \quad J$ Mol Biol 312: 927-934.

1115 Johansson J, Persson P, Lowenadler B, Robertson B, Jornvall H, Curstedt T. 1991. Canine hydrophobic 1116 surfactant polypeptide SP-C. A lipopeptide with one thioester-linked palmitoyl group. FEBS Lett 281: $1117 \quad 119-122$.

1118 Johansson J, Szyperski T, Curstedt T, Wuthrich K. 1994. The NMR structure of the pulmonary surfactant1119 associated polypeptide SP-C in an apolar solvent contains a valyl-rich a-helix. Biochemistry 33: 6015$1120 \quad 6023$

1121 Johansson J, Nilsson G, Stromberg R, Robertson B, Jornvall H, Curstedt T. 1995. Secondary structure and 1122 biophysical activity of synthetic analogues of the pulmonary surfactant polypeptide SP-C. Biochem J $1123 \quad 307: 535-541$.

1124 Johansson J. 1998. Structure and properties of surfactant protein C. Biochim Biophys Acta 1408: 161-172.

1125 Johansson J, Some M, Linderholm BM, Almlen A, Curstedt T, Robertson B. 2003. A synthetic surfactant

1126 based on a poly-Leu SP-C analog and phospholipids: effects on tidal volumes and lung gas volumes in 1127 ventilated immature newborn rabbits. J Appl Physiol 95: 2055-2063.

1128 Kairys V, Gilson MK, Luy B. 2004. Structural model for an AxxxG-mediated dimer of surfactant1129 associated protein C. Eur J Biochem 271: 2086-2092.

1130 Kauppinen JK, Moffatt DJ, Mantsch HH, Cameron DG. 1981. Fourier self-deconvolution: A method for 1131 resolving intrinsically overlapped bands. Appl Spectr 35: 271-276. 
1132 Kramer A, Wintergalen A, Sieber M, Galla HJ, Amrein M, Guckenberger R. 2000. Distribution of the

1133 surfactant-associated protein $\mathrm{C}$ within a lung surfactant model film investigated by near-field optical

1134 microscopy. Biophys $J$ 78: 458-465.

1135 Kukol A, Adams PD, Rice LM, Brunger AT, Arkin TI. 1999. Experimentally based orientational

1136 refinement of membrane protein models: A structure for the influenza A M2 H+ channel. J Mol Biol

$1137 \quad 286: 951-962$

1138 Kumar S, Nussinov R. 1999. Salt bridge stability in monomeric proteins. J Mol Biol 293: 1241-1255.

1139 Kumar S, Tsai CJ, Nussinov R. 2000. Factors enhancing protein thermostability. Protein Eng 13: 179-191.

1140 Kumar S, Nussinov R. 2002. Relationship between ion pair geometries and electrostatic strengths in

$1141 \quad$ proteins. Biophys $J$ 83: 1595-1612.

1142 Leonenko Z, Gill S, Baoukina S, Monticelli L, Doehner J, Gunasekara L, Felderer F, Rodenstein M, Eng

1143 LM, Amrein M. 2007. An elevated level of cholesterol impairs self-assembly of pulmonary surfactant

1144 into a functional film. Biophys $J$ 93: 674-683.

1145 Liang J, Naveed H, Jimenez-Morales D, Adamian L, Lin M. 2012. Computational studies of membrane 1146 proteins: Models and predictions for biological understanding. Biochim Biophys Acta 1818: 927-941.

1147 Luy B, Diener A, Hummel RP, Sturm E, Ulrich WR, Griesinger C. 2004. Structure and potential C-

1148 terminal dimerization of a recombinant mutant of surfactant-associated protein $\mathrm{C}$ in chloroform/

1149 methanol. Eur J Biochem 271: 2076-2085.

1150 Marqusee S, Baldwin RL. 1987. Helix stabilization by Glu--Lys+ salt bridges in short peptides of de novo 1151 design. Proc Natl Acad Sci USA 84: 8898-8902.

1152 Na Nakorn P, Meyer MC, Flach CR, Mendelsohn R, Galla H-J. 2007. Surfactant protein C and lung 1153 function: new insights into the role of alpha-helical length and palmitoylation. Eur Biophys $J 36$ : 477$1154 \quad 489$.

1155 Nerelius C, Fitzen M, Johansson J. 2010. Amino acid sequence determinants and molecular chaperones in 1156 amyloid fibril formation. Biochem Biophys Res Commun 396: 2-6. 
1157 Nilsson G, Gustafsson M, Vandenbussche G, Veldhuizen E, Griffiths WJ, Sjovall J, Haagsman HP,

1158 Ruysschaert J-M, Robertson B, Curstedt T, Johansson J. 1998. Synthetic peptide-containing

1159 surfactants: Evaluation of transmembrane versus amphipathic helices and surfactant protein C poly-

$1160 \quad$ valyl to poly-leucyl substitution. Eur J Biochem 255: 116-124.

1161 Nogee LM, Dunbar AE, III, Wert SE, Askin F, Hamvas A, Whitsett JA. 2001. A mutation in the surfactant

1162 protein C gene associated with familial interstitial lung disease. N Engl J Med 344: 573-579.

1163 Notter RH, Finkelstein JN, Taubold RD. 1983. Comparative adsorption of natural lung surfactant, 1164 extracted phospholipids, and artificial phospholipid mixtures to the air-water interface. Chem Phys

1165 Lipids 33: 67-80.

1166 Pastrana B, Maulone AJ, Mendelsohn R. 1991. Fourier transform infrared studies of secondary structure 1167 and orientation of pulmonary surfactant SP-C and its effect on the dynamic surface properties of 1168 phospholipids. Biochemistry 30: 10058-10064.

1169 Plasencia I, Rivas L, Keough KM, Marsh D, Perez-Gil J. 2004. The N-terminal segment of pulmonary 1170 surfactant lipopeptide SP-C has intrinsic propensity to interact with and perturb phospholipid bilayers. $1171 \quad$ Biochem J 377: 183-193.

1172 Polin RA, Carlo WA, Committee on Fetus and Newborn. 2014. Surfactant replacement therapy for 1173 preterm and term neonates with respiratory distress. Pediatrics 133: 156-163.

1174 Rajan R, Awasthi SK, Bhattacharjya S, Balaram P. 1997. "Teflon-coated peptides": hexafluoroacetone 1175 trihydrate as a structure stabilizer for peptides. Biopolymers 42: 125-128.

1176 Roccatano D, Fioroni M, Zacharias M, Colombo G. 2005. Effect of hexafluoroisopropanol alcohol on the 1177 structure of melittin: A molecular dynamics simulation study. Protein Sci 14: 2582-2589.

1178 Sarker M, Waring AJ, Walther FJ, Keough KMW, Booth V. 2007. Structure of Mini-B, a functional

1179 fragment of surfactant protein B, in detergent micelles. Biochemistry 46: 11047-11056.

1180 Schwan AL, Singh SP, Davy JA, Waring AJ, Gordon LM, Walther FJ, Wang Z, Notter RH. 2011.

1181 Synthesis and activity of a novel diether phosphonoglycerol in phospholipase-resistant synthetic $1182 \quad$ lipid:peptide lung surfactants. Medchemcomm 2: 1167-1173. 
1183 Snider C, Jayasinghe S, Hristova K, White SH. 2009. MPEx: a tool for exploring membrane proteins.

$1184 \quad$ Protein Sci 18: 2624-2628.

1185 Spragg RG, Taut FJ, Lewis JF, Schenk P, Ruppert C, Dean N, Krell K, Karabinis A, Gunther A. 2011.

1186 Recombinant Surfactant Protein C-based Surfactant for Patients with Severe Direct Lung Injury. Am $J$

1187 Respir Crit Care Med 183: 1055-1061.

1188 Takamoto DY, Lipp MM, von NA, Lee KY, Waring AJ, Zasadzinski JA. 2001. Interaction of lung

1189 surfactant proteins with anionic phospholipids. Biophys $J$ 81: 153-169.

1190 Toyoshima C, Nakasako M, Nomura H, Ogawa H. 2000. Crystal structure of the calcium pump of

1191 sarcoplasmic reticulum at 2.6 A resolution. Nature 405: 647-655.

1192 Trovato A, Chiti F, Maritan A, Seno F. 2006. Insight into the structure of amyloid fibrils from the analysis 1193 of globular proteins. PloS Comput Biol 2: e170.

1194 Trovato A, Seno F, Tosatto SC. 2007. The PASTA server for protein aggregation prediction. Protein Eng 1195 Des Sel 20: 521-523.

1196 Vandenbussche G, Clercx A, Curstedt T, Johansson J, Jornvall H, Ruysschaert JM. 1992. Structure and 1197 orientation of the surfactant-associated protein C in a lipid bilayer. Eur J Biochem 203: 201-209.

1198 von Nahmen A, Schenk M, Sieber M, Amrein M. 1997. The structure of a model pulmonary surfactant as 1199 revealed by scanning force microscopy. Biophys $J$ 72: 463-469.

1200 Voss T, Schafer KP, Nielsen PF, Schafer A, Maier C, Hannappel E, Maassen J, Landis B, Klemm K, 1201 Przybylski M. 1992. Primary structure differences of human surfactant-associated proteins isolated 1202 from normal and proteinosis lung. Biochim Biophys Acta 1138: 261-267.

1203 Walther FJ, Gordon LM, Zasadzinski JA, Sherman MA, Waring AJ. 2000. Surfactant protein B and C $1204 \quad$ analogues. Mol Genet Metab 71: 342-351.

1205 Walther FJ, Hernandez-Juviel J, Gordon LM, Waring AJ, Stenger P, Zasadzinski JA. 2005. Comparison of 1206 three lipid formulations for synthetic surfactant with a surfactant protein B analog. Exp Lung Res 31: $1207 \quad 563-579$.

1208 Walther FJ, Waring AJ, Sherman MA, Zasadzinski JA, Gordon LM. 2007a. Hydrophobic surfactant 1209 proteins and their analogues. Neonatology 91: 303-310. 
1210 Walther FJ, Waring AJ, Hernandez-Juviel J, Gordon LM, Schwan AL, Jung C-L, Chang Y, Wang Z, Notter

1211 RH. 2007b. Dynamic surface activity of a fully synthetic phospholipase-resistant lipid/peptide lung $1212 \quad$ surfactant. PLoS One 2: e1039.

1213 Walther FJ, Waring AJ, Hernandez-Juviel JM, Gordon LM, Wang Z, Jung CL, Ruchala P, Clark AP, Smith

1214 WM, Sharma S, Notter RH, 2010. Critical structural and functional roles for the N-terminal insertion

1215 sequence in surfactant protein B analogs. PLoS One 5: e8672.

1216 Walther FJ, Hernandez-Juviel JM, Gordon LM, Waring AJ. 2014. Synthetic surfactant containing SP-B

1217 and SP-C mimics is superior to single-peptide formulations in rabbits with chemical acute lung injury.

$1218 \quad$ PeerJ in press.

1219 Wang Z, Gurel O, Baatz JE, Notter RH. 1996. Acylation of pulmonary surfactant protein-C is required for 1220 its optimal surface active interactions with phospholipids. J Biol Chem 271: 19104-19109.

1221 Waring AJ, Walther FJ, Gordon LM, Hernandez-Juviel JM, Hong T, Sherman MA, Alonso C, Alig T,

1222 Braun A, Bacon D, Zasadzinski JA. 2005. The role of charged amphipathic helices in the structure and 1223 function of surfactant protein B. J Peptide Res 66: 364-374.

1224 Whitsett JA. 2002. Genetic basis of familial interstitial lung disease: misfolding or function of surfactant 1225 protein C? Am J Respir Crit Care Med 165: 1201-1202.

1226 Willander H, Askarieh G, Landreh M, Westermark P, Nordling K, Keranen H, Hermansson E, Hamvas A, 1227 Nogee LM, Bergman T, Saenz A, Casals C, Aqvistg J, Jornvall H, Berglund H, Presto J, Knight SD, 1228 Johansson J. 2012. High-resolution structure of a BRICHOS domain and its implications for anti1229 amyloid chaperone activity on lung surfactant protein C. Proc Natl Acad Sci U S A 109: 2325-2329.

1230 Willson DF, Thomas NJ, Tamburro R, Truemper E, Truwit J, Conaway M, Traul C, Egan EE, Pediatric $1231 \quad$ Acute Lung and Sepsis Investigators Network. 2013. Pediatric calfactant in acute respiratory distress 1232 syndrome trial. Pediatr Crit Care Med 14: 657-665.

1233 Wimley WC, Creamer TP, White SH. 1996. Solvation energies of amino acid side chains and backbone in 1234 a family of host-guest pentapeptides. Biochemistry 35: 5109-5124. 
1235 Wimley WC, Gawrisch K, Creamer TP, White SH. 1996. Direct measurement of salt-bridge solvation

1236 energies using a peptide model system: implications for protein stability. Proc Natl Acad Sci U S A 93:

$1237 \quad 2985-2990$.

1238 Yamaguchi S, Huster D, Waring AJ, Lehrer RI, Kearney WR, Tack BF, Hong M. 2001. Orientation and 1239 dynamics of an antimicrobial peptide in the lipid bilayer by solid-state NMR spectroscopy. Biophys $J$ $1240 \quad 81: 2203-2214$

1241 Yamaguchi S, Hong T, Waring A, Lehrer RI, Hong M. 2002. Solid-state NMR investigations of peptide-

1242 lipid interaction and orientation of a beta-sheet antimicrobial peptide, protegrin. Biochemistry 41:

$1243 \quad 9852-9862$.

1244 Zandomeneghi G, Krebs MRH, McCammon MG, Fandrich M. 2004. FTIR reveals structural differences 1245 between native beta-sheet proteins and amyloid fibrils. Protein Sci 13: 3314-3321.

1246

1247

1248

1249 


\section{Table 1 (on next page)}

Proportions of secondary structure and maximum tilt angles of the membrane $\alpha$-helix for SP-C peptide mimics in synthetic surfactant lipids.

Proportions of secondary structure ${ }^{\mathrm{a}}$ and maximum tilt angles $(\Theta)$ of the membrane $\alpha$-helix ${ }^{b}$ for SP-C peptide mimics in synthetic surfactant lipids with $10 \mathrm{mM}$ deuterated phosphate buffer ( $\mathrm{pD} 7.4$ ), as estimated from the ATR-FTIR spectra of the peptide amide I band. 


\begin{tabular}{llllll}
\hline System & $\begin{array}{l}\text { \% } \\
\text { Conformation }\end{array}$ & & & & \multicolumn{1}{c}{$\begin{array}{l}\text { Max. Tilt } \\
\text { Angle }^{\mathbf{b}}\end{array}$} \\
\hline & a-helix & $\beta$-sheet & loop-turn & disordered & $\theta$ \\
DPPC:POPC:POP & & & & & \\
G & & & & & \\
SP-Cff & 5.6 & 74.3 & 2.9 & 17.2 & -- \\
SP-Cff ion-lock 1 & 44.6 & 17.8 & 15.7 & 22.0 & $34.2^{\circ}$ \\
SP-CSS & 24.5 & 58.6 & 9.1 & 7.9 & -- \\
SP-CSS ion-lock 1 & 63.1 & 5.5 & 25.5 & 5.9 & $22.6^{\circ}$ \\
SP-C33 & 67.2 & 6.9 & 13.9 & 12.0 & $22.5^{\circ}$ \\
SP-C33 ion-lock 1 & 66.9 & 3.6 & 21.7 & 7.8 & $22.7^{\circ}$ \\
SP-C33 ion-lock 2 & 65.1 & 7.7 & 18.0 & 9.18 & $23.2^{\circ}$ \\
\hline
\end{tabular}




\section{Table 2 (on next page)}

Proportions of secondary structure for SP-C peptide mimics in hexafluoroisopropanol buffer.

Proportions of secondary structure for SP-C peptide mimics in $10 \mathrm{mM}$ deuterated phosphate buffer ( $\mathrm{pD} 7.4$ ) and $35 \%$ hexafluoroisopropanol (HFIP)/65\% dPBS, as estimated from the ATR-FTIR spectra of the peptide amide I band 


\begin{tabular}{lllll}
\hline System & \% Conformation & & & \\
\hline & $\alpha$-helix & $\beta$-sheet & loop-turn & disordered \\
dPBS & & & & \\
SP-Cff & 7.4 & 51.9 & 24.0 & 10.6 \\
SP-Cff ion-lock 1 & 17.8 & 2.9 & 59.9 & 19.5 \\
SP-CsS & 15.8 & 49.7 & 14.6 & 19.9 \\
SP-CSS ion-lock 1 & 40.8 & 11.8 & 18.8 & 28.7 \\
SP-C33 UCLA & 50.0 & 14.4 & 17.2 & 18.4 \\
SP-C33 ion-lock 1 & 57.8 & 14.8 & 12.2 & 15.1 \\
35\% HFIP/dPBS & & & & \\
SP-Cff & 19.3 & 53.2 & 17.8 & 9.7 \\
SP-Cff ion-lock 1 & 53.0 & 13.9 & 16.9 & 16.1 \\
SP-CSS & 18.5 & 35.6 & 22.1 & 23.9 \\
SP-CSS ion-lock 1 & 51.1 & 10.4 & 33.1 & 14.7 \\
SP-C33 UCLA & 52.6 & 14.1 & 18.6 & 13.7 \\
SP-C33 ion-lock 1 & 49.6 & 8.01 & 1.42 & \\
\hline
\end{tabular}




\section{Figure 1}

Amino-acid sequences (1-letter codes) for native SP-C proteins and SP-C mimic peptides.

Human, dog and pig SP-C are the native sequences (35 or 34 residues), with palmitoyl groups (not shown) attached to the Cys residues in the N-terminal domain. SP-C mimics are based on the native sequences, except that the palmitoylcysteines have been replaced by Phe or Ser residues. The SP-C mimic sequences have been slightly shifted to permit maximal overlaps with the SP-C protein sequences. rSP-C is a 35-residue, recombinant form of human SP-C, with Phe residues replacing Cys-4 and Cys-5 and lle replacing Met-32; SPC33 UCLA has the same sequence as the conventional SP-C33; SP-Cff ion-lock 1 is SP$\mathrm{Cff}(\mathrm{E} 20 / \mathrm{K} 24)$, SP-Css ion-lock 1 is SP-Css(E20/K24) and SP-C33 ion-lock 1 is SPC33(E20/K24), each with an ion-lock between Glu--20 and Lys ${ }^{+}-24$. SP-C33 ion-lock 2 is SPC33(K15/E19; E20/K24), with ion-locks between Lys'-15 and Glu-19 and between Glu'-20 and Lys $^{+}-24$. Paired glutamates and lysines are underlined red residues, with salt-bridges represented as red lines. The SP-Cff and SP-Css mimics are each 34-residues long (except for $\mathrm{rSP}-\mathrm{C}$ ) and based on the native SP-C class, while those based on the SP-C33 class are 33-residues. 
Amino-acid sequences of Human, Dog and Pig SP-C proteins and SP-C mimics

\begin{tabular}{|c|c|c|c|c|}
\hline SP-C Protein or Mimic & \multicolumn{4}{|c|}{ Amino-acid sequence } \\
\hline \multicolumn{5}{|l|}{ SP-C proteins } \\
\hline Numbering & 1 & 10 & 20 & 30 \\
\hline Human SP-C & FGIPCCPVHL & KRLLIVVVVV & VLIVVVIVGA & LLMGL \\
\hline Dog SP-C & GIPCFPSSL & KRLLIIVVVI & VLVVVVIVGA & LLMGL \\
\hline Pig SP-C & LRIPCCPVNL & KRLLVVVVVV & VLVVVVIVGA & LLMGL \\
\hline \multicolumn{5}{|l|}{$S P-C$ mimics } \\
\hline & 1 & 10 & 20 & 30 \\
\hline rSP-C (Venticute $®)$ & FGIPFFPVHL & KRLLIVVVVV & VLIVVVIVGA & LLIGL \\
\hline SP-Cff & GIPFFPVHL & KRLLIVVVVV & VLIVVVIVGA & LLMGL \\
\hline SP-Cff ion-lock 1 & GIPFFPVHL & KRLLIVVVVV & $\frac{E}{l} L I V K V I V G A$ & LLMGL \\
\hline SP-Css & GIPSSPVHL & KRLLIVVVVV & VLIVVVIVGA & LLMGL \\
\hline \multirow[t]{2}{*}{ SP-Css ion-lock 1} & GIPSSPVHL & KRLLIVVVVV & $\underline{\underline{E} L I V \underline{K V I V G A}}$ & LLMGL \\
\hline & 1 & 10 & 20 & 30 \\
\hline SP-C33 UCLA & IPSSPVHLK & RLKLLLLLLL & LILLLILGAL & LMGL \\
\hline SP-C33 ion-lock 1 & IPSSPVHLK & RLKLLLLLLL & $\underset{I}{\text { IILLKILGAL }}$ & LMGL \\
\hline SP-C33 ion-lock 2 & IPSSPVHLK & RLKLLKLLLLE & $\frac{E}{\mid} I L L K I L G A L$ & LMGL \\
\hline
\end{tabular}




\section{Figure 2}

FTIR spectra of the amide I bands for SP-C mimic peptides in surfactant lipids (DPPC:POPC:POPG 5:3:2).

Plate A: SP-Css is the dashed red line, SP-Css ion-lock 1 is the solid red-line, SP-Cff is the dashed black line and SP-Cff ion-lock 1 is the solid black line. The spectra for either SP-Css or SP-Cff in surfactant lipids with 10 deuterated $\mathrm{mM}$ phosphate buffer (pD 7.4) principally show $\beta$-sheet, with a major peak at $1630-1631 \mathrm{~cm}^{-1}$ and a very low companion peak at $\sim 1691$ $\mathrm{cm}^{-1}$. The SP-Css and SP-Cff spectra also show significant shoulders at $\sim 1653-1657 \mathrm{~cm}^{-1}$, indicating minor $\alpha$-helical structures. The corresponding spectra for SP-Css ion-lock 1 and SP-Cff ion-lock 1 in surfactant lipids primarily show $\alpha$-helix with maxima at $\sim 1653-1657 \mathrm{~cm}^{-1}$, indicating that incorporation of the Glu- Lys ${ }^{+}$ion-pair in either SP-C mimic effectively reverses their $\beta$-sheet properties.Plate B: SP-C33 UCLA is a solid magenta line, SP-C33 ion-lock 1 is a solid green line, and SP-C33 ion-lock 2 is a solid blue line. The spectra for SP-C33 UCLA and the SP-C33 ion-locks in lipids all closely overlap, with shared maxima at $\sim 1655 \mathrm{~cm}^{-}$ ${ }^{1}$ indicating predominate $\alpha$-helix. The introduction of a single or double Glu- Lys ${ }^{+}$ion-pair in SP-C33 does not raise the $\alpha$-helical levels over that seen with the parent SP-C33 UCLA (Table 1). Peptide concentrations were $470 \mu \mathrm{M}$, and sequences are in Fig. 1. FTIR spectra for peptide in lipid mixtures (Fig. 2A and B) were obtained by subtracting the lipid spectrum hydrated with $10 \mathrm{mM}$ deuterated phosphate buffer ( $\mathrm{pD}$ 7.4) from those of peptides in lipid with $10 \mathrm{mM}$ phosphate buffer (pD 7.4). 

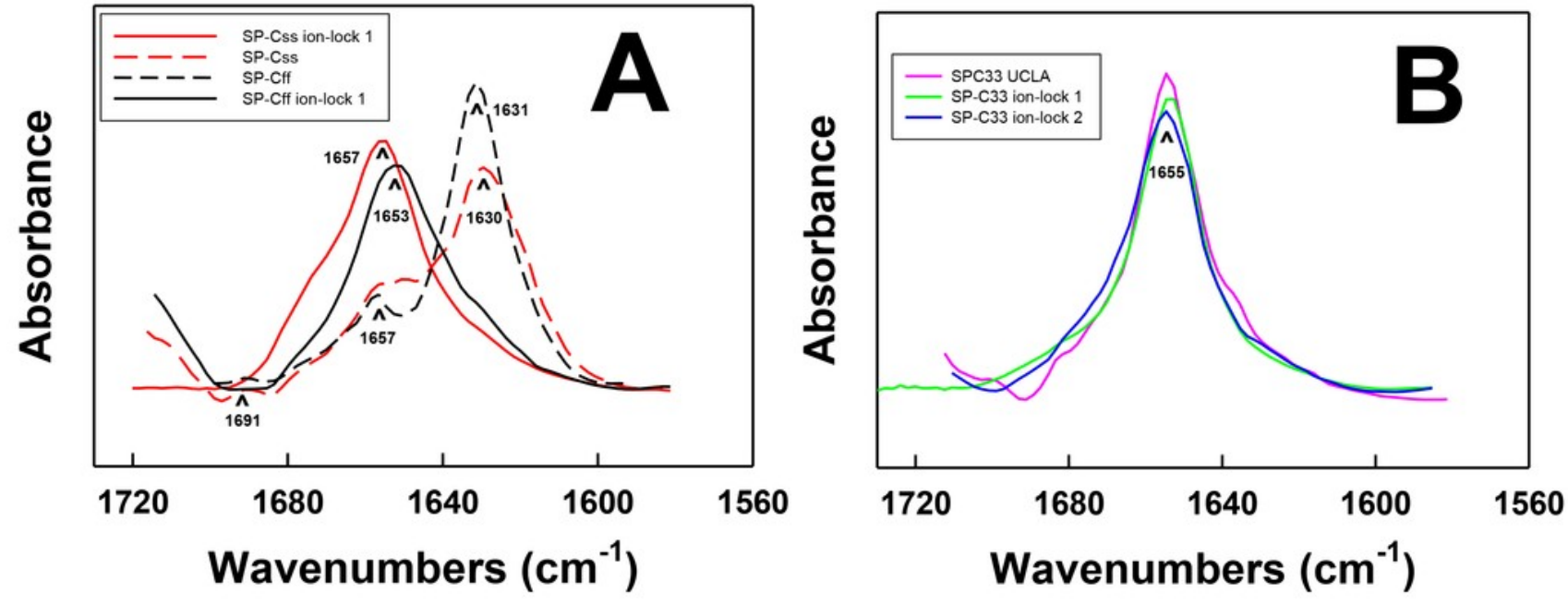


\section{Figure 3}

Plot of $\alpha$-helix (\%) from FTIR spectra of SP-C peptides in surfactant lipids versus relative PASTA energies.

Percent (\%) $\alpha$-helix was determined from the FTIR spectra of SP-Css, SP-Cff, SP-Css ionlock 1, SP-Cff ion-lock 1, SP-C33 UCLA, SP-C33 ion-lock 1 and SP-C33 ion-lock 2 (Fig. 2; Table 1). Relative energy values were from PASTA, with less negative values reflecting a lower propensity to form $\beta$-sheet. The PASTA energies for SP-Css, SP-Cff, SP-Css ion-lock 1 and SP-Cff ion-lock 1 are calculated from the C-terminal residues 7-27, while those from SP-C33, SP-C33 ion-lock 1 and SP-C33 ion-lock 2 are from the C-terminal segment 6-26. The sequences and numbering for the SP-C peptides are in Fig. 1. 


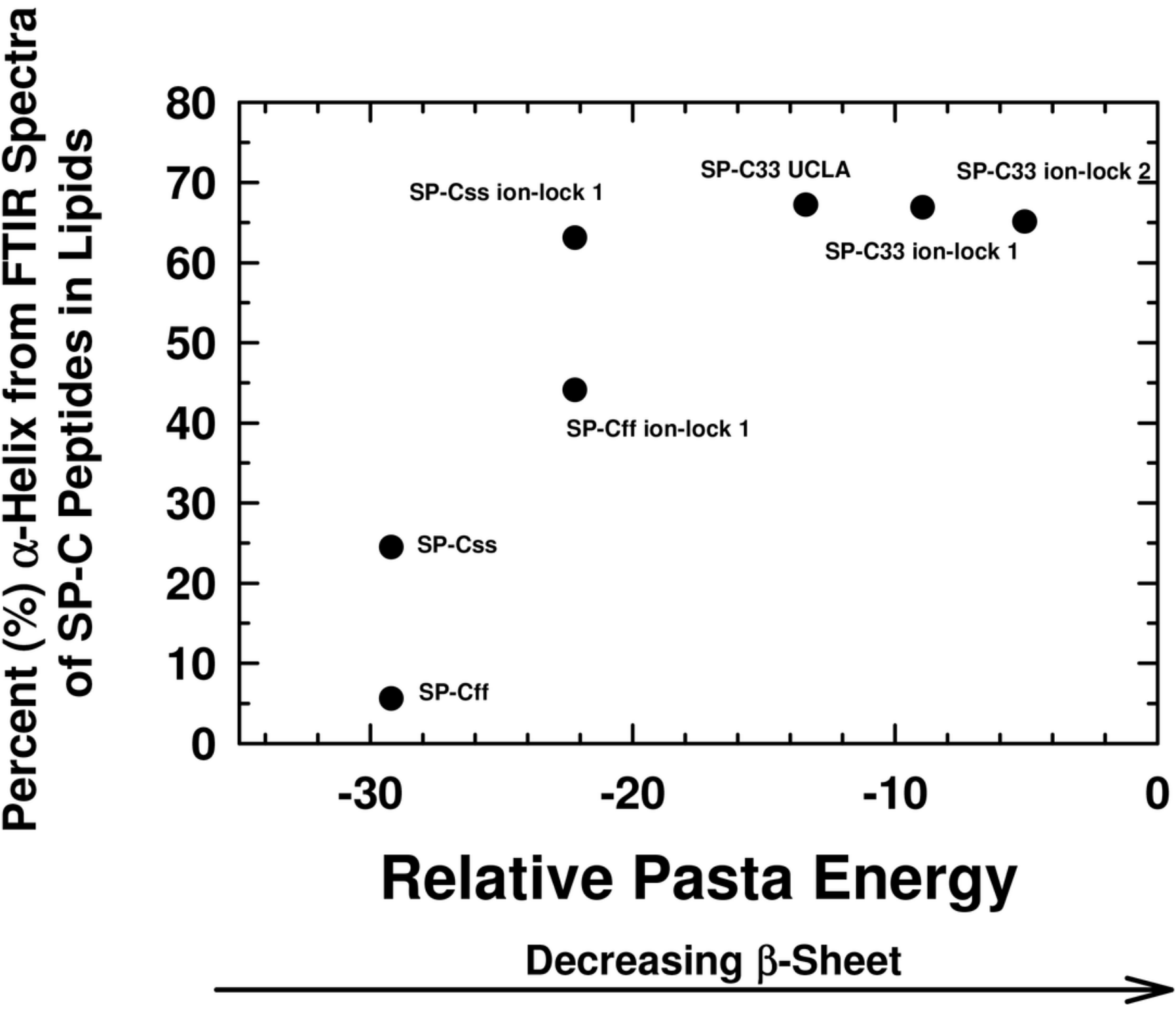




\section{Figure 4}

Plot of hydropathy $(\mathrm{kcal} / \mathrm{mol})$ vs. relative PASTA energy values, determined for the rSPC, SP-Css ion-lock 1, SP-C33 and SP-C33 ion-lock 1 and SP-C33 ion-lock 2 peptides.

Hydropathy $(\mathrm{kcal} / \mathrm{mol})$ is a measure of the hydrophobic partitioning for helical peptides into transmembrane environments, determined using MPEx. Positive hydropathy predicts elevated lipid binding for helical peptides, while more negative values forecast greater watersolubility. Relative energy values were from PASTA, with less negative values reflecting a lower propensity to form $\beta$-sheet. Top-Left Quadrant: The rSP-C (filled circle) and SP-Css ion-lock 1 (open circles) values are shown. The PASTA energies for rSP-C and SP-Css ionlock 1 are calculated from the respective segments 7-27, while MPEx hydropathies are determined from the respective segments 12-30. The SP-Css ion-lock 1 value, connected to the rSP-C value by a solid line, reflects the hydropathy for an $\alpha$-helical peptide with an engaged salt-bridge (i.e., close-range) between Glu--Lys+' Alternatively, the SP-Css ion-lock 1 value, connected to the SP-C value by a dashed line, indicates a hydropathy with a disengaged salt-bridge (i.e., long-range). The SP-Cff ion-lock 1 values overlap those of SPCss ion-lock 1. SP-Css and SP-Cff points are not included, because MPEx does not accurately predict the transmembrane insertion of such short helices (i.e., $\leq \sim 8$ residues) (Table 1). Top-Right Quadrant: The SP-C33 (filled inverted triangles) and SP-C33 ion-lock 1 and 2 (open inverted triangles) values are shown. The PASTA energies for SP-C33, SP-C33 ion-lock 1 and SP-C33 ion-lock 2 are each calculated from the C-terminal segment 6-26, while MPEx hydropathy is determined from each 13-31 segment. The SP-C33 ion-lock values, connected to the parent SP-C33 value by a solid line, reflect the hydropathies of $\alpha$ helical peptides with engaged salt-bridges between the Glu- $\mathrm{Lys}^{+}$pair. Alternatively, the SPC33 ion-lock values, connected to the SP-C33 value by dashed lines, indicate hydropathies with disengaged salt-bridges between the Glu--Lys ${ }^{+}$pairs. See Figure 1 for sequences. 


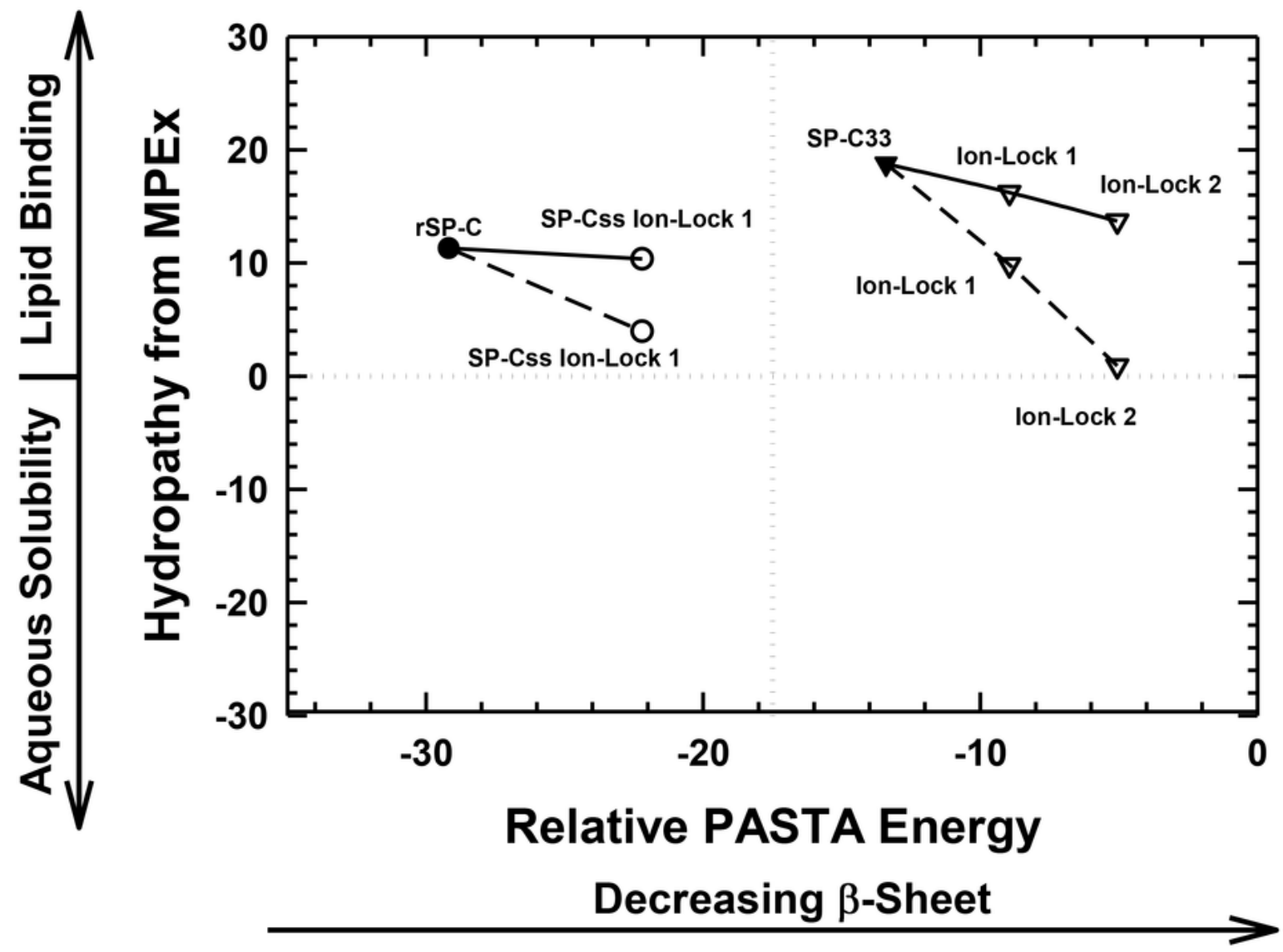




\section{Figure 5}

ATR-FTIR polarized spectra of the amide I band for synthetic SP-C peptide mimics in oriented DPPC:POPC:POPG (5:3:2) bilayers with $10 \mathrm{mM}$ deuterated phosphate buffer (pD 7.4).

The bottom spectra in each plate were recorded with parallel (para; solid line) and perpendicular (perp; dashed line) polarized light with respect to the incident plane. The dichroic spectra at top in each plate is the difference between the spectrum recorded with parallel polarized light from that obtained with perpendicular polarized light. Recorded and subtracted spectra for each SP-C peptide are drawn using the same ordinate scale. Plate A: SP-Css ion-lock 1. Plate B: SP-Cff ion-lock 1. Plate C: SP-C33 ion-lock 1. Plate D: SP-C33 UCLA. See Figure 1 for SP-C mimic sequences.
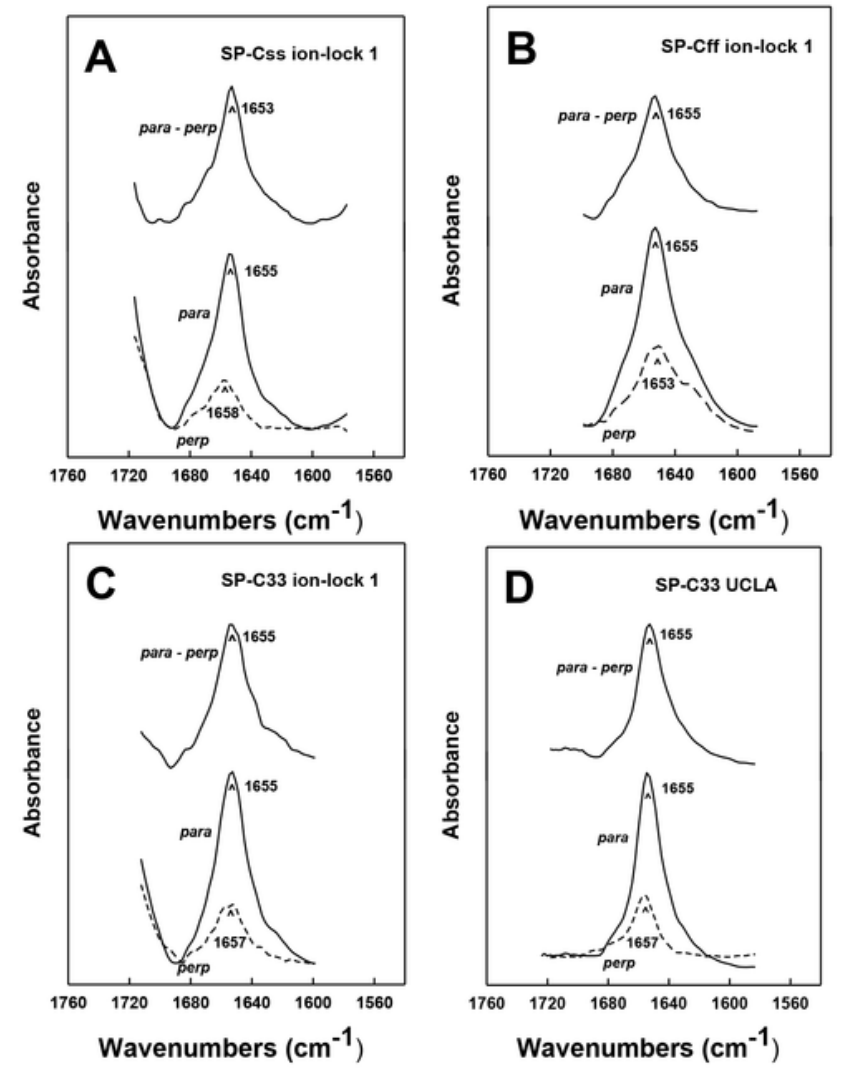


\section{Figure 6}

The percent (\%) unexchanged hydrogens vs. time ( $\mathrm{min}$ ) from FTIR of SP-C mimics in surfactant lipids.

SP-Css ion-lock 1 (inverted triangle, green), SP-C33 UCLA (square, magenta) and SP-Cff ion-lock 1 (hex, blue) were each incorporated into DPPC:POPC:POPG (5:3:2, weight ratio), and the peptide-lipid sample on the ATR crystal was initially hydrated by bubbling nitrogen through a $10 \mathrm{mM}$ deuterated phosphate buffer $(\mathrm{pD} 7.4)$ at $37^{\circ} \mathrm{C}$. The time-dependent H/D exchange of the amide group was determined from the decay of the amide II band area $\left(1525-1565 \mathrm{~cm}^{-1}\right)$ with respect to the corresponding amide I band area $\left(\sim 1600-1700 \mathrm{~cm}^{-1}\right)$ as a function of time $(0-6 \mathrm{~h})$.

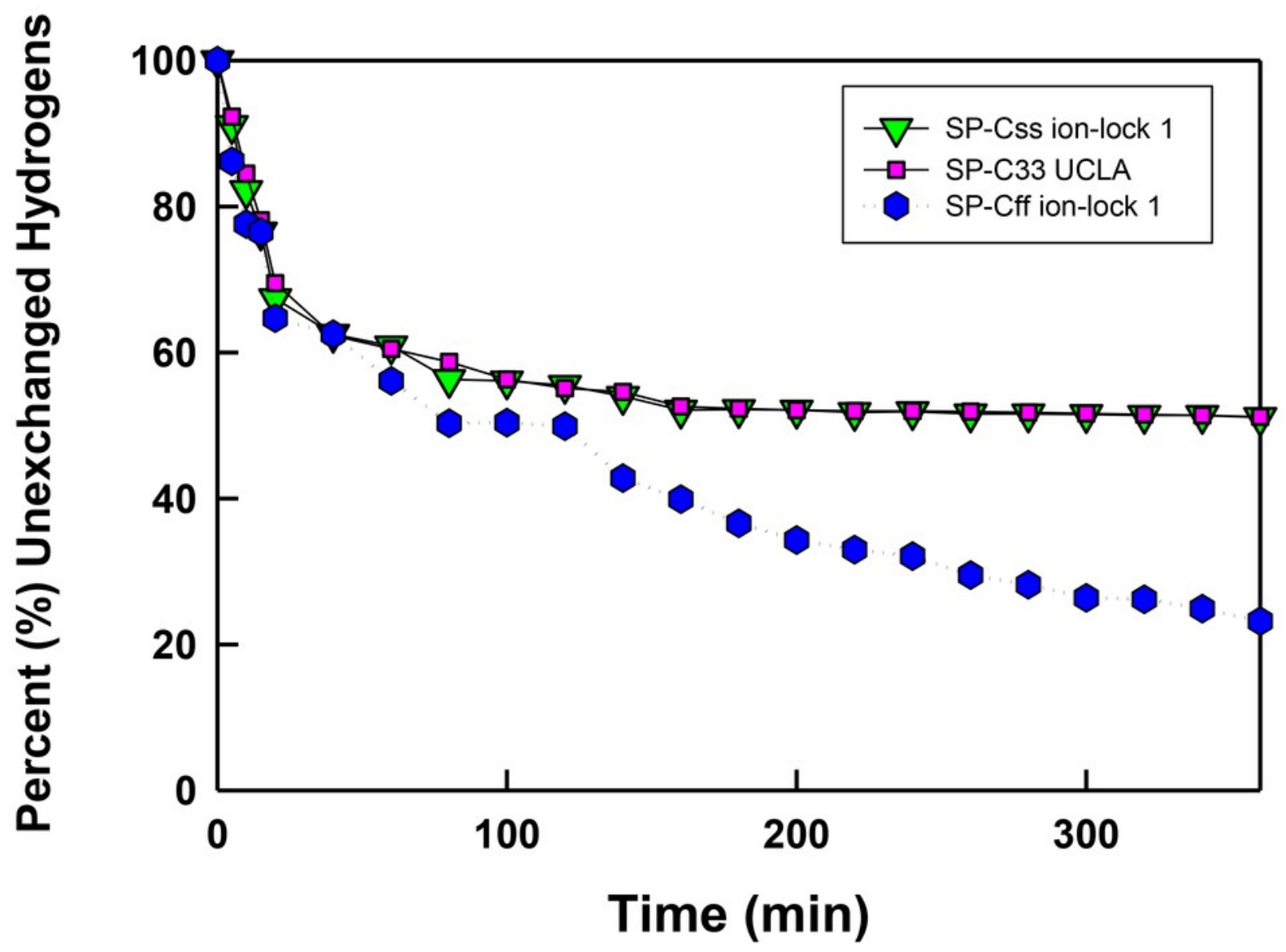




\section{Figure 7}

Adsorption of synthetic surfactant compositions containing SP-Cff, SP-C33 and SP-C ion-lock mimics plus synthetic phospholipids (5:3:2 weight ratio DPPC:POPC:POPG).

Adsorption was measured as Adsorption Surface Pressure $(\mathrm{mN} / \mathrm{m})$, following injection of a surfactant bolus beneath the interface of a stirred subphase at time zero. Surface pressure is the amount of surface tension lowering below that of the pure subphase (normal saline adjusted to $\mathrm{pH} 7.0$ ) at $37 \pm 0.5^{\circ} \mathrm{C}$. Higher surface pressure equates to lower surface tension. Surfactant concentration was $0.0625 \mathrm{mg}$ phospholipid/ml of subphase. Data are Means \pm SEM for $n=4$. Sequences for SP-Cff, SP-Css ion-lock 1, SP-Cff ion-lock 1, SP-C33 ion-lock 2 and SP-C33 UCLA are given in Figure 1.
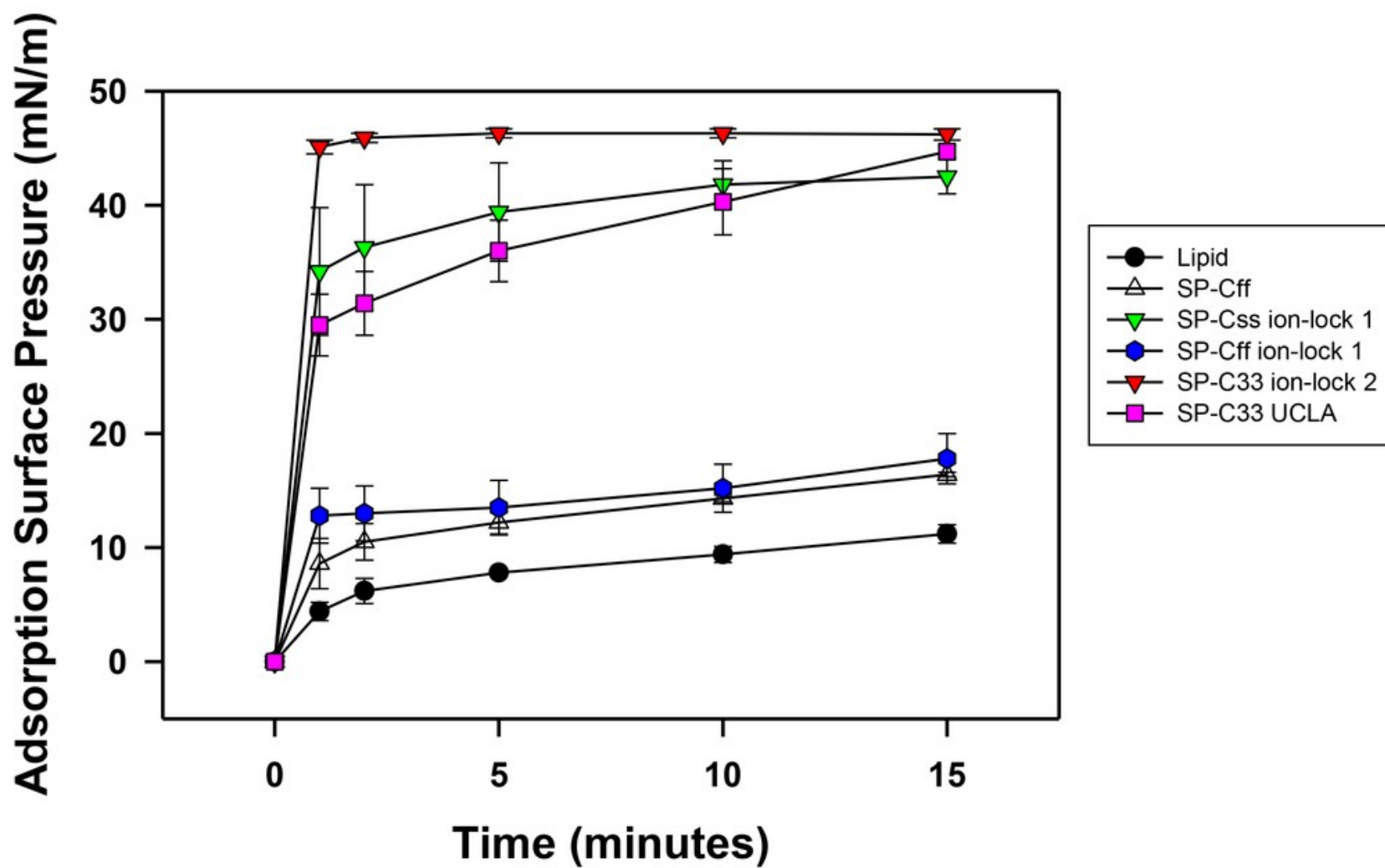


\section{Figure 8}

Surface activity of synthetic surfactants on the captive bubble surfactometer.

Minimum and maximum surface tension values are plotted for synthetic lipids (Lipids) with $3.0 \%$ by weight SP-Cff, SP-Css, SP-Css ion-lock 1, SP-C33 ion-lock 1, SP-Cff ion-lock 1 and SP-C33 UCLA (positive control) or synthetic lipids alone (negative control) for 10 successive compression cycles on a captive bubble surfactometer $\left(20 \mathrm{cycles} / \mathrm{min}, 37^{\circ} \mathrm{C}\right)$. Synthetic lipids = 5:3:2 (weight ratio) DPPC:POPC:POPG. Values shown are means with estimated SEM, for $\mathrm{n}=4-6$. See Figure 1 for SP-C mimic sequences.
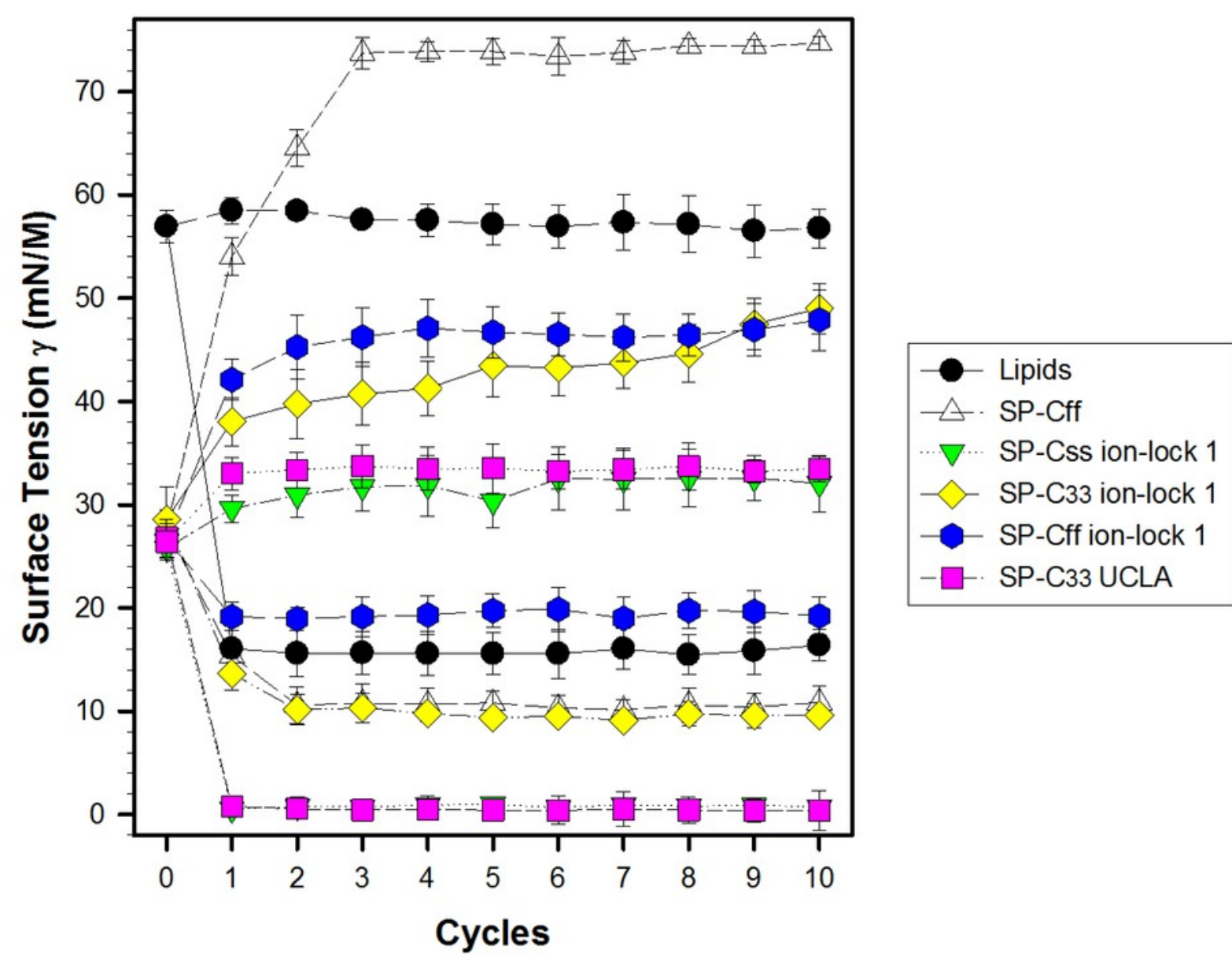


\section{Figure 9}

Arterial oxygenation and dynamic compliance in surfactant-treated, ventilated rabbits with ARDS induced by in vivo lavage.

Panel A: Arterial partial pressure of oxygen $\left(\mathrm{PaO}_{2}\right.$ in torr) shown as a function of time for groups of at least 4 rabbits treated with synthetic lung surfactants containing synthetic lipids with $3.0 \%$ by weight SP-Cff, SP-Css ion-lock 1, SP-C33 ion-lock 1, SP-Cff ion-lock 1, and SP-C33 UCLA (positive control), or synthetic lipids alone (negative control). Synthetic lipids are DPPC:POPC:POPG (5:3:2, weight ratio). Panel B: Dynamic compliance $\left(\mathrm{mL} / \mathrm{kg} / \mathrm{cm} \mathrm{H}_{2} \mathrm{O}\right)$

shown as a function of time for groups of at least 4 rabbits treated with the same lung surfactant preparations as shown in panel A. Data are shown as means $\pm S E M$, for $n=4-7$. Sequences for SP-C mimics are shown in Figure 1.

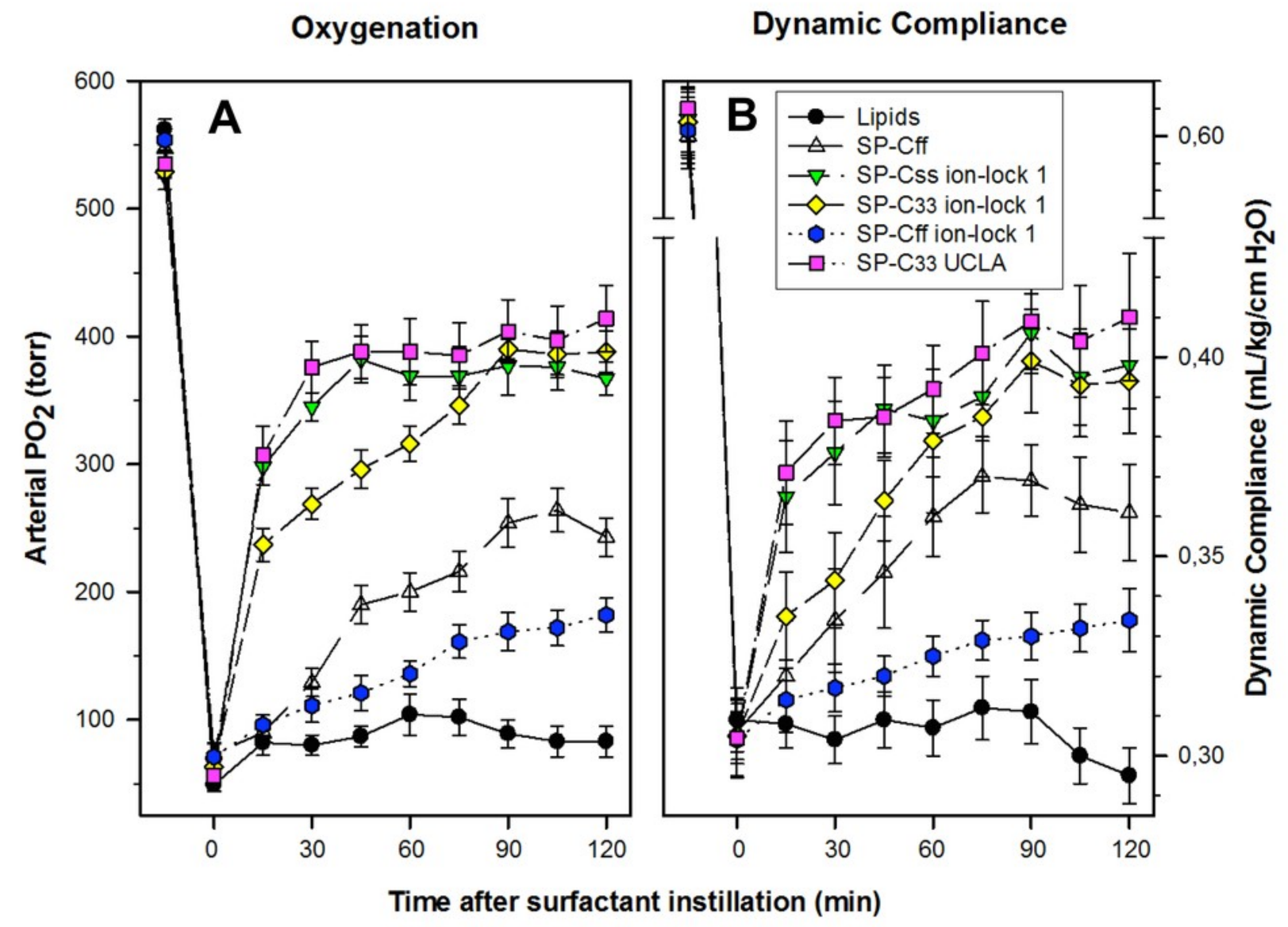

